\title{
ON THE CLASSIFICATION OF FAKE LENS SPACES
}

\author{
TIBOR MACKO, CHRISTIAN WEGNER
}

\begin{abstract}
In the first part of the paper we present a classification of fake lens spaces of dimension $\geq 5$ whose fundamental group is the cyclic group of order any $N \geq 2$. The classification is stated in terms of the simple structure sets in the sense of surgery theory. The results use and extend the results of Wall and others in the cases $N=2$ and $N$ odd and the results of the authors of the present paper in the case $N=2^{K}$.

In the second part we study the suspension map between the simple structure sets of lens spaces of different dimensions. As an application we obtain an inductive geometric description of the torsion invariants of fake lens spaces.
\end{abstract}

\section{INTRODUCTION}

A fake lens space is the orbit space of a free action of a finite cyclic group $G$ on a sphere $S^{2 d-1}$. It is a generalization of the notion of a lens space which is the orbit space of a free action which comes from a unitary representation. The classification of lens spaces is a classical topic in algebraic topology and algebraic $K$-theory well explained for example in Mil66]. For the classification of fake lens spaces in dimension $\geq 5$ methods of surgery theory are especially suitable. The classification of fake lens spaces with $G$ of order $N=2$ or $N$ odd was obtained and published in the books Wal99, LdM71]. In [MW08 we addressed the problem for $N=2^{K}$. In the present paper we treat the general case $N \geq 2$. The previous classification results are used as an input.

The general approach to solving the classification problem is the same in all cases. Firstly, Reidemeister torsion is used to provide the simple homotopy classification. The homeomorphism classification within a simple homotopy type can then be formulated in terms of the simple structure set $\mathcal{S}^{s}(X)$ of a closed $n$-manifold $X$. An element of $\mathcal{S}^{s}(X)$ is represented by a simple homotopy equivalence $f: M \rightarrow X$ from a closed $n$-manifold $M$. Two such $f: M \rightarrow X, f^{\prime}: M^{\prime} \rightarrow X$ are equivalent if there exists a homeomorphism $h: M \rightarrow M^{\prime}$ such that $f^{\prime} \circ h \simeq f$. The simple structure set $\mathcal{S}^{s}(X)$ is a priori just a pointed set with the base point id: $X \rightarrow X$. However, it can also be endowed with a preferred structure (in some sense) of an abelian group (see [Ran92, chapter 18]). Surgery theory is the standard method for calculating the simple structure set. In the case of fake lens spaces, in addition to the usual methods of surgery theory, the so-called reduced $\rho$-invariant and a certain amount of calculations with this invariant are needed.

We have recalled the above scheme in MW08 in detail and we have made the calculations in the case $N=2^{K}$. In the general case we follow the same scheme, but different methods and calculations are needed in certain stages, in particular those concerning the reduced $\rho$-invariant. Also the results for $N=2^{K}$ and $N$ odd are used as an input. That is the reason why we present them in a separate paper. Therefore when discussing the results of the general scheme we will often refer the

2000 Mathematics Subject Classification. Primary: 57R65, 57S25.

Key words and phrases. lens space, structures set, $\rho$-invariant, normal invariants, surgery.

The authors are supported by SFB 478 Geometrische Strukturen in der Mathematik, Münster. 
reader for a detailed discussion to [MW08 and here we will concentrate on the calculational issues that are different for general $N \geq 2$.

The invariants which distinguish the elements of this set and the homeomorphism classification of polarized fake lens spaces rather than just elements of the simple structure set are also discussed. See Introduction and section 1 of MW08 for a more detailed general discussion on the relations between these classification problems.

In the second part of the paper we study the suspension map between the simple structure sets of lens spaces of different dimensions. As an application we obtain an inductive geometric description of the invariants of fake lens spaces on the torsion subgroups of the simple structure sets calculated in the first part.

\section{Statement of the Results}

Definition 1.1. A fake lens space $L^{2 d-1}(\alpha)$ is a manifold obtained as the orbit space of a free action $\alpha$ of the group $G=\mathbb{Z}_{N}$ on $S^{2 d-1}$.

The fake lens space $L^{2 d-1}(\alpha)$ is a $(2 d-1)$-dimensional manifold with $\pi_{1}\left(L^{2 d-1}(\alpha)\right) \cong$ $G=\mathbb{Z}_{N}$ and universal cover $S^{2 d-1}$. The main theorem in this paper is:

Theorem 1.2. Let $L^{2 d-1}(\alpha)$ be a fake lens space with $\pi_{1}\left(L^{2 d-1}(\alpha)\right) \cong \mathbb{Z}_{N}$ where $N=2^{K} \cdot M$ with $K \geq 0, M$ odd and $d \geq 3$. Then we have

$$
\mathcal{S}^{s}\left(L^{2 d-1}(\alpha)\right) \cong \bar{\Sigma}_{N}(d) \oplus \bigoplus_{i=1}^{c} \mathbb{Z}_{2^{\min \{K, 1\}}} \oplus \bigoplus_{i=1}^{c} \mathbb{Z}_{2^{\min \{K, 2 i\}}}
$$

where $\bar{\Sigma}_{N}(d)$ is a free abelian group. If $N$ is odd then its rank is $(N-1) / 2$. If $N$ is even then its rank is $N / 2-1$ if $d=2 e+1$ and $N / 2$ if $d=2 e$. In the torsion summand we have $c=\lfloor(d-1) / 2\rfloor$.

The isomorphism from the above theorem has an interpretation in terms of known invariants. The first invariant is the reduced $\rho$-invariant, denoted $\widetilde{\rho}$, which takes values in $\mathbb{Q} R_{\widehat{G}}^{(-1)^{d}}$, the underlying abelian group of the $(-1)^{d}$-eigenspace of the rationalized complex representation ring of $G$ modulo the ideal generated by the regular representation. The group $\bar{\Sigma}_{N}(d)$ is a subgroup of this group. The invariants $\mathbf{r}_{2 i}$ below are closely related to the normal invariants from surgery theory, see the discussion from [MW08, section 1] for more details.

Corollary 1.3. Let $L^{2 d-1}(\alpha)$ be a fake lens space with $\pi_{1}\left(L^{2 d-1}(\alpha)\right) \cong \mathbb{Z}_{N}$ where $N=2^{K} \cdot M$ with $K \geq 0, M$ odd and $d \geq 3$. There exists a collection of invariants

$$
\mathbf{r}_{4 i}: \mathcal{S}^{s}\left(L^{2 d-1}(\alpha)\right) \longrightarrow \mathbb{Z}_{2^{\min \{K, 2 i\}}} \quad \text { and } \quad \mathbf{r}_{4 i-2}: \mathcal{S}^{s}\left(L^{2 d-1}(\alpha)\right) \longrightarrow \mathbb{Z}_{2^{\min \{K, 1\}}}
$$

where $1 \leq i \leq c=\lfloor(d-1) / 2\rfloor$ which together with the $\widetilde{\rho}$-invariant induce a one-toone correspondence between elements $a \in \mathcal{S}^{s}\left(L^{2 d-1}(\alpha)\right)$ and

(1) $\tilde{\rho}(a) \in \bar{\Sigma} \subset \mathbb{Q} R_{\widehat{G}}^{(-1)^{d}}$

(2) $\mathbf{r}_{2 i}(a) \in \mathbb{Z}_{2^{\min \{K, 1\}}}, \mathbb{Z}_{2^{\min \{K, 2 i\}}}$

To obtain classification of fake lens spaces rather than classification of elements of the simple structure set we follow Wall [Wal99, chapter 14E] and work with polarized fake lens spaces. These are fake lens spaces equipped with a choice of orientation and a choice of a generator of the fundamental group, see MW08, Definition 2.2]. The simple homotopy type of a polarized fake lens space $L^{2 d-1}(\alpha)$ is given uniquely by its Reidemeister torsion $\Delta\left(L^{2 d-1}(\alpha)\right)$, which is a unit in $\mathbb{Q} R_{G}$, the rational group ring of $G$ modulo the ideal generated by the norm element, as described in [MW08, Proposition 2.3]. That means that for two polarized fake lens spaces $L^{2 d-1}(\alpha)$ and $L^{2 d-1}(\beta)$ with the fundamental group $G$ there is a simple homotopy equivalence $f_{\alpha, \beta}: L^{2 d-1}(\alpha) \rightarrow L^{2 d-1}(\beta)$ of polarized fake lens spaces 
unique up to homotopy if and only if the Reidemeister torsions of $L^{2 d-1}(\alpha)$ and $L^{2 d-1}(\beta)$ coincide. The simple homotopy equivalence $f_{\alpha, \beta}$ gives us an element of the simple structure set $\mathcal{S}^{s}\left(L^{2 d-1}(\beta)\right)$. We can formulate the classification as follows:

Corollary 1.4. Let $L^{2 d-1}(\alpha)$ and $L^{2 d-1}(\beta)$ be polarized lens spaces with the fundamental group $G=\mathbb{Z}_{N}$, where $N=2^{K} \cdot M$ with $K \geq 0, M$ odd and $d \geq 3$. There exists a polarized homeomorphism between $L^{2 d-1}(\alpha)$ and $L^{2 d-1}(\beta)$ if and only if

(1) $\Delta\left(L^{2 d-1}(\alpha)\right)=\Delta\left(L^{2 d-1}(\beta)\right)$,

(2) $\rho\left(L^{2 d-1}(\alpha)\right)=\rho\left(L^{2 d-1}(\beta)\right)$,

(3) $\mathbf{r}_{2 i}\left(f_{\alpha, \beta}\right)=0$ for all $i$.

In the second part of the paper we study the so-called suspension homomorphisms $\Sigma: \mathcal{S}^{s}\left(L^{2 d-1}\left(\alpha_{k}\right)\right) \rightarrow \mathcal{S}^{s}\left(L^{2 d+1}\left(\alpha_{k}\right)\right)$, see MW08, section 3.3]. Here $\alpha_{k}$ denotes certain actions of $\mathbb{Z}_{N}$ on $S^{2 d-1}$ and on $S^{2 d+1}$ which yield standard lens spaces, see definition in [MW08, section 2]. The reason for restricting to the case $\alpha=\alpha_{k}$ is explained in Remark 7.7. The main results are Theorem 6.1 and 6.2 , Their statements require certain notation and are therefore relegated to section 6 . Nevertheless, we can now state a corollary of these two theorems.

Corollary 1.5. Let $N=2^{K} \cdot M$ with $K \geq 1, M$ odd. If $e \geq 1$ then there is an exact sequence:

$$
0 \rightarrow \mathcal{S}^{s}\left(L^{4 e+1}\left(\alpha_{k}\right)\right) \stackrel{\Sigma}{\rightarrow} \mathcal{S}^{s}\left(L^{4 e+3}\left(\alpha_{k}\right)\right) \rightarrow \mathbb{Z} \rightarrow 0 .
$$

If $e \geq 2$ then there is an exact sequence

$$
0 \rightarrow \mathbb{Z} \rightarrow \mathcal{S}^{s}\left(L^{4 e-1}\left(\alpha_{k}\right)\right) \stackrel{\Sigma}{\rightarrow} \mathcal{S}^{s}\left(L^{4 e+1}\left(\alpha_{k}\right)\right) \rightarrow \mathbb{Z}_{2} \rightarrow 0 .
$$

As an application we obtain an improvement on the invariants from Corollary 1.4. Although the invariants $\mathbf{r}_{4 i-2}$ have certain geometric description, which we recall in Remark 7.6, we are not able to give such a description for the invariants $\mathbf{r}_{4 i}$. However, using our results about the suspension map we obtain new invariants $\overline{\mathbf{r}}_{4 i}$. Although, as explained in section [7, there is still a certain choice involved, the invariants $\overline{\mathbf{r}}_{4 i}$ are an improvement, since after that choice is made, they have an inductive geometric interpretation. A more detailed statement again needs some notation and is therefore given in section 7 as Corollary 7.3 , and Remark 7.5.

The paper is organized as follows. In section 2 we recall the information about the simple structure set of fake lens spaces which we obtain from surgery theory. In section 3 we recall the definition of the $\widetilde{\rho}$-invariant and we prove some of its properties, in particular we show that it gives a group homomorphism of the simple structure set. In section 4 we recall the results in the cases $N=2^{K}$ and $N=M$ odd which are used in section 5, which contains the calculations. In section 6 the suspension map is studied and in the final section 7 we provide the geometric description of the torsion invariants.

\section{THE SURGERY EXACT SEQUENCE}

For the simple homotopy classification of fake lens spaces we refer the reader to [MW08, section 2] where we review the results of Wall. Here we concentrate on the homeomorphism classification within a simple homotopy type. The main tool is the surgery exact sequence computing the structure set $\mathcal{S}^{s}(X)$ for a given $n$-manifold $X$ with $n \geq 5$ :

$$
\cdots \rightarrow \mathcal{N}_{\partial}(X \times I) \stackrel{\theta}{\rightarrow} L_{n+1}^{s}(G) \stackrel{\partial}{\rightarrow} \mathcal{S}^{s}(X) \stackrel{\eta}{\rightarrow} \mathcal{N}(X) \stackrel{\theta}{\rightarrow} L_{n}^{s}(G),
$$

where $G=\pi_{1}(X)$. The terms in the sequence are reviewed in the detail in MW08, section 3]. Now we analyze them for $X=L_{N}^{2 d-1}(\alpha)$. The following proposition from HT00 describes the $L$-theory needed. Here $R_{\mathbb{C}}(G)$ denotes the complex 
representation ring of a group $G$ and the superscripts \pm denote the \pm -eigenspaces with respect to the involution given by complex conjugation.

Theorem 2.1. For $G=\mathbb{Z}_{N}$ we have that

$$
\begin{gathered}
L_{n}^{s}(G) \cong \begin{cases}4 \cdot R_{\mathbb{C}}^{+}(G) & n \equiv 0(\bmod 4)(\text { G-sign, purely real) } \\
0 & n \equiv 1(\bmod 4) \\
4 \cdot R_{\overparen{C}}^{-}(G) \oplus \mathbb{Z}_{2} & n \equiv 2(\bmod 4)(\text { G-sign, purely imaginary, Arf }) \\
\mathbb{Z}_{2} & n \equiv 3(\bmod 4)(\text { codimension } 1 \text { Arf })\end{cases} \\
\widetilde{L}_{2 k}^{s}(G) \cong 4 \cdot R_{\widehat{G}}^{(-1)^{k}} \text { where } R_{\widehat{G}}^{(-1)^{k}} \text { is } R_{\mathbb{C}}^{(-1)^{k}}(G) \text { modulo the regular representation } .
\end{gathered}
$$

For the normal invariants we have in general

Corollary 2.2. If $X$ is rationally trivial we have an isomorphism of abelian groups

$$
\begin{aligned}
\mathcal{N}(X) & \cong \mathcal{N}(X)_{(2)} \oplus \mathcal{N}(X)_{(\text {odd })} \\
& \cong\left(\bigoplus_{i \geq 1}\left(H^{4 i}\left(X ; \mathbb{Z}_{(2)}\right) \oplus H^{4 i-2}\left(X ; \mathbb{Z}_{2}\right)\right)\right) \oplus K O(X) \otimes \mathbb{Z}\left[\frac{1}{2}\right]
\end{aligned}
$$

When $X$ is a fake lens space $L^{2 d-1}(\alpha)$ with $\pi_{1}\left(L^{2 d-1}(\alpha)\right) \cong G=\mathbb{Z}_{N}$ we obtain

$$
\begin{aligned}
\mathcal{N}\left(L^{2 d-1}(\alpha)\right) \cong & \bigoplus_{i=1}^{\lfloor(d-1) / 2\rfloor} H^{4 i}\left(L^{2 d-1}(\alpha) ; \mathbb{Z}_{(2)}\right) \oplus \bigoplus_{i=1}^{\lfloor d / 2\rfloor} H^{4 i-2}\left(L^{2 d-1}(\alpha) ; \mathbb{Z}_{2}\right) \\
& \oplus K O\left(L^{2 d-1}(\alpha)\right) \otimes \mathbb{Z}\left[\frac{1}{2}\right]
\end{aligned}
$$

The first two summands can be easily calculated explicitly and we denote the factors

$$
\begin{aligned}
\mathbf{t}_{4 i} & : \mathcal{N}\left(L^{2 d-1}(\alpha)\right) \rightarrow H^{4 i}\left(L^{2 d-1}(\alpha) ; \mathbb{Z}_{(2)}\right) \cong \mathbb{Z}_{2^{K}} \\
\mathbf{t}_{4 i-2} & : \mathcal{N}\left(L^{2 d-1}(\alpha)\right) \rightarrow H^{4 i-2}\left(L^{2 d-1}(\alpha) ; \mathbb{Z}_{2}\right) \cong \mathbb{Z}_{2} .
\end{aligned}
$$

The last summand is more difficult to calculate explicitly, but we will not need the exact calculation. Note that when Wall analyzed the case $N=M$ he only needed that the order of the group $K O\left(L^{2 d-1}(\alpha)\right) \otimes \mathbb{Z}\left[\frac{1}{2}\right]$ is $M^{c}$ with $c=\lfloor(d-1) / 2\rfloor$, which is an easy Atiyah-Hirzebruch spectral sequence argument. This will also be sufficient for us. We will still need some more notation, so the projection onto this last summand will be denoted

$$
\mathbf{t}_{(o d d)}: \mathcal{N}\left(L^{2 d-1}(\alpha)\right) \rightarrow K O\left(L^{2 d-1}(\alpha)\right) \otimes \mathbb{Z}\left[\frac{1}{2}\right] .
$$

We will also sometimes put together the 2-local invariants and denote $\mathbf{t}_{(2)}=\left(\mathbf{t}_{2 i}\right)_{i}$ and finally $\mathbf{t}=\left(\mathbf{t}_{(2)}, \mathbf{t}_{(\text {odd })}\right)$. These projections will also be sometimes used to identify the elements of $\mathcal{N}\left(L^{2 d-1}(\alpha)\right)$ as $t=\left(\left(t_{2 i}\right)_{i}, t_{(\text {odd })}\right)$. Even more information is obtained from the following

Theorem 2.3 (Wal99).

(1) If $d=2 e$ then the map

$$
\theta: \mathcal{N}\left(L^{2 d-1}(\alpha)\right) \rightarrow L_{2 d-1}^{s}(G)=L_{4 e-1}^{s}(G)=\mathbb{Z}_{2}
$$

is given by $\theta(x)=\mathbf{t}_{4 e-2}(x) \in \mathbb{Z}_{2}$.

(2) The map

$$
\theta: \mathcal{N}_{\partial}\left(L^{2 d-1}(\alpha) \times I\right) \rightarrow L_{2 d}^{s}(G)
$$

maps onto the summand $L_{2 d}^{s}(1)$. 
Hence we obtain the short exact sequence

$$
0 \rightarrow \widetilde{L}_{2 d}^{s}(G) \stackrel{\partial}{\rightarrow} \mathcal{S}^{s}\left(L^{2 d-1}(\alpha)\right) \stackrel{\eta}{\rightarrow} \widetilde{\mathcal{N}}\left(L^{2 d-1}(\alpha)\right) \rightarrow 0
$$

where

$$
\begin{aligned}
& \tilde{\mathcal{N}}\left(L^{4 e-1}(\alpha)\right)=\operatorname{ker}\left(\mathbf{t}_{4 e-2}: \mathcal{N}\left(L^{4 e-1}(\alpha)\right) \rightarrow H^{4 e-2}\left(L^{4 e-1}(\alpha) ; \mathbb{Z}_{2}\right) \cong \mathbb{Z}_{2}\right), \\
& \widetilde{\mathcal{N}}\left(L^{4 e+1}(\alpha)\right)=\mathcal{N}\left(L^{4 e+1}(\alpha)\right) .
\end{aligned}
$$

in other words

$$
\tilde{\mathcal{N}}\left(L^{2 d-1}(\alpha)\right) \cong \bigoplus_{i=1}^{c} \mathbb{Z}_{2^{K}} \oplus \bigoplus_{i=1}^{c} \mathbb{Z}_{2} \oplus K O\left(L^{2 d-1}(\alpha)\right) \otimes \mathbb{Z}\left[\frac{1}{2}\right]
$$

where $c=\lfloor(d-1) / 2\rfloor$ and where the order of the last summand is $M^{c}$. The first term in the sequence (2.5) is understood by Theorem 2.1, the third term is understood by (2.6). Hence we are left with an extension problem.

Remark 2.4. We will also need to work with fake complex projective spaces. Wall's calculation of $\mathcal{S}\left(\mathbb{C} P^{d-1}\right)$ and $\mathcal{N}\left(\mathbb{C} P^{d-1}\right)$ is reviewed in [MW08, subsection 3.1]. Recall that $\mathcal{S}\left(\mathbb{C} P^{d-1}\right)$ is a subgroup of $\mathcal{N}\left(\mathbb{C} P^{d-1}\right)$. Both groups are calculated in terms of invariants $\mathbf{s}_{4 i} \in \mathbb{Z}$ and $\mathbf{s}_{4 i-2} \in \mathbb{Z}_{2}$.

In this paper we will also make systematic use of the functoriality of the normal invariants and of the structure set. Let $H<G \leq S^{1}$ be an inclusion of subgroups and let $\alpha$ be a free action of $G$ on $S^{2 d-1}$. The inclusion $H<G$ induces a free action of $H$ which we also denote $\alpha$. Let now $L_{G}(\alpha)$ denote the resulting $(2 d-1)$ dimensional fake lens spaces in case $G<S^{1}$ or the resulting $(2 d-2)$-dimensional fake complex projective space in case $G=S^{1}$. We have fiber bundles

$$
p_{H}^{G}: L_{H}(\alpha) \longrightarrow L_{G}(\alpha) .
$$

The maps $p_{H}^{G}$ induce the vertical maps in the following diagram

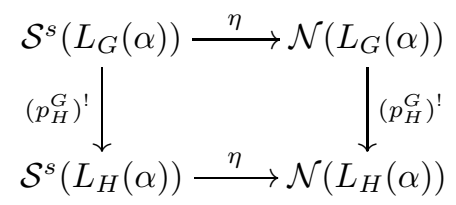

The right hand map preserves the localization at 2 and away from 2 and we have that $\left(p_{H}^{G}\right)^{!}: \mathcal{N}\left(L_{G}(\alpha)\right)_{(2)} \longrightarrow \mathcal{N}\left(L_{H}(\alpha)\right)_{(2)}$ is given by reduction modulo $|H|$. Suppose now that $G=\mathbb{Z}_{N}, N=2^{K} \cdot M, M$ odd, and $H=\mathbb{Z}_{N^{\prime}}, N^{\prime}=2^{K^{\prime}} \cdot M^{\prime}, M^{\prime}$ odd. If $M=M^{\prime}$ then $\left(p_{H}^{G}\right)^{!}: \mathcal{N}\left(L_{G}(\alpha)\right)_{(\text {odd })} \longrightarrow \mathcal{N}\left(L_{H}(\alpha)\right)_{(\text {odd })}$ is an isomorphism. If $K=K^{\prime}$ then $\left(p_{H}^{G}\right)^{!}: \mathcal{N}\left(L_{G}(\alpha)\right)_{(2)} \longrightarrow \mathcal{N}\left(L_{H}(\alpha)\right)_{(2)}$ is an isomorphism. In fact we have for $N=2^{K} \cdot M$ with $M$ odd that

$$
p_{2^{K}}^{N} \oplus p_{M}^{N}: \mathcal{N}\left(L_{N}\right) \cong \mathcal{N}\left(L_{N}\right)_{(2)} \oplus \mathcal{N}\left(L_{N}\right)_{(\text {odd })} \longrightarrow \mathcal{N}\left(L_{2^{K}}\right) \oplus \mathcal{N}\left(L_{M}\right)
$$

is an isomorphism. (We have left out $(\alpha)$ everywhere due to the lack of space.) In the sequel we will sometimes use the notation $L_{G}(\alpha)$ when we feel the need for specifying $G$. In the other cases the group involved should be clear.

Recall from [MW08, section 2] the definition of the actions $\alpha_{k}$ of $\mathbb{Z}_{N}$ on $S^{2 d-1}$ for $k \in \mathbb{N},(k, N)=1$, which yield standard lens spaces $L^{2 d-1}\left(\alpha_{k}\right)$. Further recall from [MW08, subsection 3.3] the join of fake lens spaces. The join with $L^{1}\left(\alpha_{k}\right)$ defines a homomorphism $\Sigma_{k}: \mathcal{S}^{s}\left(L^{2 d-1}\left(\alpha_{1}\right)\right) \longrightarrow \mathcal{S}^{s}\left(L^{2 d+1}\left(\alpha_{k}\right)\right)$. The inclusion $L^{2 d-1}\left(\alpha_{1}\right) \subset$ $L^{2 d+1}\left(\alpha_{k}\right)$ induces a restriction map on the groups of normal invariants denoted by 
res : $\mathcal{N}\left(L^{2 d+1}\left(\alpha_{k}\right)\right) \longrightarrow \mathcal{N}\left(L^{2 d-1}\left(\alpha_{1}\right)\right)$ and we have a commutative diagram Wal99, Lemma 14A.3]:

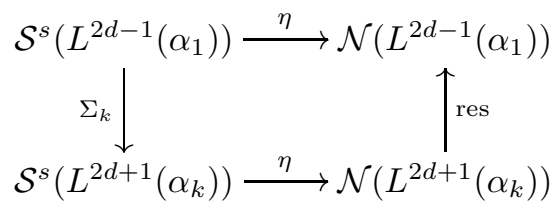

Note that we have $t_{2 i}=\operatorname{res}\left(t_{2 i}\right)$. Moreover, the map

$$
\text { res: } \tilde{\mathcal{N}}\left(L^{2 d+1}\left(\alpha_{1}\right)\right) \longrightarrow \tilde{\mathcal{N}}\left(L^{2 d-1}\left(\alpha_{1}\right)\right)
$$

is an isomorphism when $d=2 e+1$ and it is onto when $d=2 e$. A similar diagram exists for the situation $\mathbb{C} P^{d}=\mathbb{C} P^{d-1} *$ pt.

\section{THE $\rho$-INVARIANT}

Similarly as in [MW08, we will use the $\rho$-invariant of odd-dimensional manifolds to solve our extension problem. The definition we use is the same as in MW08, but the formulation of the properties and their proofs have to be adjusted to the more general case. This is the content of the present section.

\subsection{Definitions.}

Recall the definition of the $\rho$-invariant (see also [MW08, subsection 4.1]).

Definition 3.1. AS68, Remark after Corollary 7.5] Let $X^{2 d-1}$ be a closed manifold with $\pi_{1}(X) \cong G$ a finite group. Define

$$
\rho(X)=\frac{1}{k} \cdot \mathrm{G}-\operatorname{sign}(\tilde{Y}) \in \mathbb{Q} R^{(-1)^{d}}(G) /\langle\mathrm{reg}\rangle
$$

for some $k \in \mathbb{N}$ and $(Y, \partial Y)$ such that $\pi_{1}(Y) \cong \pi_{1}(X)$ and $\partial Y=k \cdot X$. The symbol $\langle\mathrm{reg}\rangle$ denotes the ideal generated by the regular representation.

We remind the reader that there is also another definition which works for actions of compact Lie groups, in particular for $S^{1}$-actions, on certain odd-dimensional manifolds. Whenever the two definitions apply, they coincide. For $G<S^{1}$ we identify $R(G)$ with $\mathbb{Z} \widehat{G}$ and we adopt the notation $R_{\widehat{G}}:=R(G) /\langle\operatorname{reg}\rangle$ and $R_{\widehat{G}}=$ $\mathbb{Z}[\chi] /\left\langle 1+\chi+\cdots+\chi^{N-1}\right\rangle$ as explained in [MW08, section 4.1].

Hence we have the $\rho$-invariant defined for fake lens spaces and for fake complex projective spaces. We continue with a list of some basic properties. For the join $L * L^{\prime}$ of fake lens spaces $L$ and $L^{\prime}$ we have [Wal99, chapter 14A]

$$
\rho\left(L * L^{\prime}\right)=\rho(L) \cdot \rho\left(L^{\prime}\right) .
$$

For $L^{1}\left(\alpha_{k}\right)$ we have Wal99, Proof of Theorem 14C.4]

$$
\rho\left(L^{1}\left(\alpha_{k}\right)\right)=f_{k} \in \mathbb{Q} R_{\widehat{G}}^{-}
$$

where $f_{k}$ is defined as follows.

Definition 3.2. For $k \in \mathbb{N}$ with $(N, k)=1$ we set

$$
f_{k}:=\frac{1+\chi^{k}}{1-\chi^{k}}
$$

and

$$
f_{k}^{\prime}:= \begin{cases}\frac{1-\chi+\chi^{2}-\cdots-\chi^{k-2}+\chi^{k-1}}{1+\chi+\chi^{2}+\cdots+\chi^{k-2}+\chi^{k-1}} & \text { for } k \text { odd }, \\ \frac{\chi^{k}-\chi^{k+1}+\chi^{k+2}-\cdots-\chi^{N-2}+\chi^{N-1}}{1+\chi+\chi^{2}+\cdots+\chi^{k-2}+\chi^{k-1}} & \text { for } k \text { even. }\end{cases}
$$

We abbreviate $f:=f_{1}$. Note that if $k$ is even, we necessarily have $N=M$ odd. 
Lemma 3.3. Let $G=\mathbb{Z}_{N}$ with $N=2^{K} \cdot M, M$ odd. For $k \in \mathbb{N}$ with $(N, k)=1$ we have

$$
f_{k} \in \mathbb{Q} R_{\widehat{G}}^{-}, \quad f_{k}=f \cdot f_{k}^{\prime}, \quad f_{k}^{\prime} \in R_{\widehat{G}} .
$$

Proof. Notice that $1-\chi^{k}$ is invertible in $\mathbb{Q} R_{\widehat{G}}$ because

$$
\left(1-\chi^{k}\right)^{-1}=-\frac{1}{N}\left(1+2 \cdot \chi^{k}+3 \cdot \chi^{2 k}+\cdots+N \cdot \chi^{(N-1) k}\right) \in \mathbb{Q} R_{\widehat{G}} .
$$

Therefore, $f_{k} \in \mathbb{Q} R_{\widehat{G}}$ and the identity

$$
\frac{1+\chi^{-k}}{1-\chi^{-k}}=-\frac{1+\chi^{k}}{1-\chi^{k}}=-f_{k}
$$

implies $f_{k} \in \mathbb{Q} R_{\widehat{G}}^{-}$. An easy calculation shows $f_{k}=f \cdot f_{k}^{\prime}$. That $f_{k}^{\prime} \in R_{\widehat{G}}$ follows from the fact that $1+\chi+\chi^{2}+\cdots+\chi^{k-1}$ is invertible in $R_{\widehat{G}}$. The inverse is given by $1+\chi^{k}+\chi^{2 k}+\cdots+\chi^{(r-1) k}$ where $r$ denotes a natural number such that $r \cdot k-1$ is a multiple of $N$.

Remark 3.4. Recall that for $G<S^{1}$ we have a canonical isomorphism $R(G)=\mathbb{Z} \widehat{G}$. Suppose that we also have a subgroup $H$ of $G$ and denote the inclusion $i: H \hookrightarrow G$. Then the ring homomorphism $R(G) \rightarrow R(H)$ induced by the restriction is identified with the ring homomorphism $\mathbb{Z} \widehat{G} \rightarrow \mathbb{Z} \widehat{H}$ induced by the group homomorphism $\widehat{i}: \widehat{G} \rightarrow \widehat{H}$. This homomorphism sends a generator to a generator. The choice of a generator $\chi$ of $\widehat{G}$ also gives us an identification $R(G)=\mathbb{Z} \widehat{G}=\mathbb{Z}[\chi] /\left\langle\chi^{|G|}-1\right\rangle$. Hence we can think of the induced homomorphism $R(G) \longrightarrow R(H)$ as of the obvious quotient map $\mathbb{Z}[\chi] /\left\langle\chi^{|G|}-1\right\rangle \longrightarrow \mathbb{Z}[\chi] /\left\langle\chi^{|H|}-1\right\rangle$.

\subsection{Homomorphism.}

The $\rho$-invariant defines a function of $\mathcal{S}^{s}(X)$ by sending $a=[h: M \longrightarrow X]$ to $\widetilde{\rho}(a)=\rho(M)-\rho(X)$. If we put on $\mathcal{S}^{s}(X)$ the abelian group structure from Ran92, chapter 18] it is not clear whether $\widetilde{\rho}$ is a homomorphism in general. It is the aim of the present subsection to show this claim for $X=L^{2 d-1}(\alpha)$. More precisely we prove the following

Proposition 3.5. There is the following commutative diagram of abelian groups and homomorphisms with exact rows

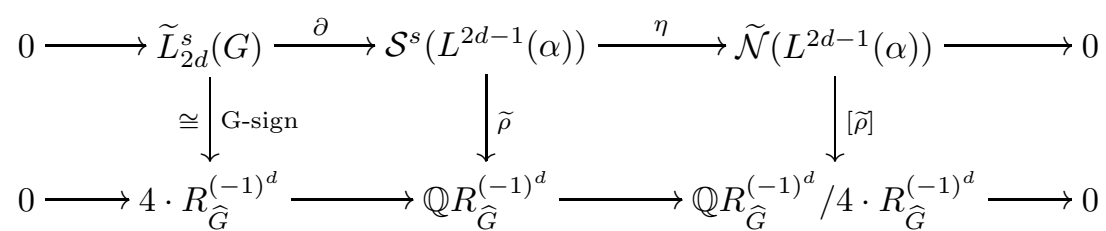

where $[\widetilde{\rho}]$ is the homomorphism induced by $\widetilde{\rho}$.

Similarly as in MW08 the commutativity of the left hand square follows from Pet70, Theorem 2.3]. We need to show that $\widetilde{\rho}$ and $[\widetilde{\rho}]$ are homomorphisms. This will be proved first for $\alpha_{1}$, then for $\alpha_{k}$, and finally for general $\alpha$.

We start with a useful lemma.

Lemma 3.6. Let $N=2^{K} \cdot M, M$ odd, $G=\mathbb{Z}_{N}$. Then there exists $g \in \mathbb{Q} R_{\widehat{G}}^{-}$such that for all $x \in \mathbb{Q} R_{\widehat{G}}^{-}$we have $x=g \cdot f \cdot x$. 
Proof. We use the Chinese remainder theorem which tell us that we have an isomorphism of rings

$$
\begin{aligned}
\mathbb{Q}[\chi] /\langle 1+\chi & \left.+\cdots+\chi^{N-1}\right\rangle \stackrel{\cong}{\longrightarrow} \\
& \cong \bigoplus_{l=0}^{K-1} \mathbb{Q}[\chi] /\left\langle 1+\chi^{2^{l}}\right\rangle \oplus \mathbb{Q}[\chi] /\left\langle 1+\left(\chi^{2^{K}}\right)+\cdots+\left(\chi^{2^{K}}\right)^{M-1}\right\rangle
\end{aligned}
$$

Denote the projections $\operatorname{pr}_{l}: \mathbb{Q}[\chi] /\left\langle 1+\chi+\cdots+\chi^{N-1}\right\rangle \longrightarrow \mathbb{Q}[\chi] /\left\langle 1+\chi^{2^{l}}\right\rangle$ and pr: $\mathbb{Q}[\chi] /\left\langle 1+\chi+\cdots+\chi^{N-1}\right\rangle \longrightarrow \mathbb{Q}[\chi] /\left\langle 1+\left(\chi^{2^{K}}\right)+\cdots+\left(\chi^{2^{K}}\right)^{M-1}\right\rangle$.

If $K=0$ then $f$ is invertible since

$$
(1+\chi)^{-1}=\left(1+\chi^{2}+\cdots+\chi^{M-2}\right) \in \mathbb{Z}[\chi] /\left\langle 1+\chi+\cdots \chi^{N-1}\right\rangle .
$$

If $K>0$ and $l>0$ then notice that $(1+\chi)$ is invertible in $\mathbb{Q}[\chi] /\left\langle 1+\chi^{2^{l}}\right\rangle$ :

$$
h_{l}:=(1+\chi)^{-1}=\frac{1}{2} \cdot A_{l} \text { with } A_{l}:=1-\chi+\chi^{2}-\chi^{3}+\cdots-\chi^{2^{l}-1} .
$$

It is also invertible in $\mathbb{Q}[\chi] /\left\langle 1+\left(\chi^{2^{K}}\right)+\cdots+\left(\chi^{2^{K}}\right)^{M-1}\right\rangle$ :

$$
h:=(1+\chi)^{-1}=-\frac{A_{K}}{M} \cdot\left(1-2 \cdot\left(\chi^{2^{K}}\right)+3 \cdot\left(\chi^{2^{K}}\right)^{2}-\cdots+M \cdot\left(\chi^{2^{K}}\right)^{M-1}\right) .
$$

Let $g \in \mathbb{Q} R_{\widehat{G}}^{-}$be such that $\operatorname{pr}_{l}(g)=h_{l}$ for $l>0, \operatorname{pr}(g)=h$ and $\operatorname{pr}_{0}(g)=0$. Further recall that we can write $x \in \mathbb{Q} R_{\widehat{G}}^{-}$as

$$
x=\sum_{r=1}^{N / 2-1} a_{r} \cdot\left(\chi^{r}-\chi^{N-r}\right) .
$$

with $a_{r} \in \mathbb{Q}$. Since $\chi^{r}-\chi^{N-r}$ is a multiple of $1+\chi$, we conclude $\operatorname{pr}_{0}(x)=0$. We obtain $\operatorname{pr}_{l}(g f x)=\operatorname{pr}_{l}(x)$ for $l>0, \operatorname{pr}(g f x)=\operatorname{pr}(x)$ and $\operatorname{pr}_{0}(g f x)=0=\operatorname{pr}_{0}(x)$. Hence $g f x=x$.

Proposition 3.7. The function $[\widetilde{\rho}]: \mathcal{N}\left(L_{N}^{2 d-1}\left(\alpha_{1}\right)\right) \longrightarrow \mathbb{Q} R_{\widehat{G}}^{(-1)^{d}} / 4 \cdot R_{\widehat{G}}^{(-1)^{d}}$ is a homomorphism.

Proof. Consider the following commutative diagram:

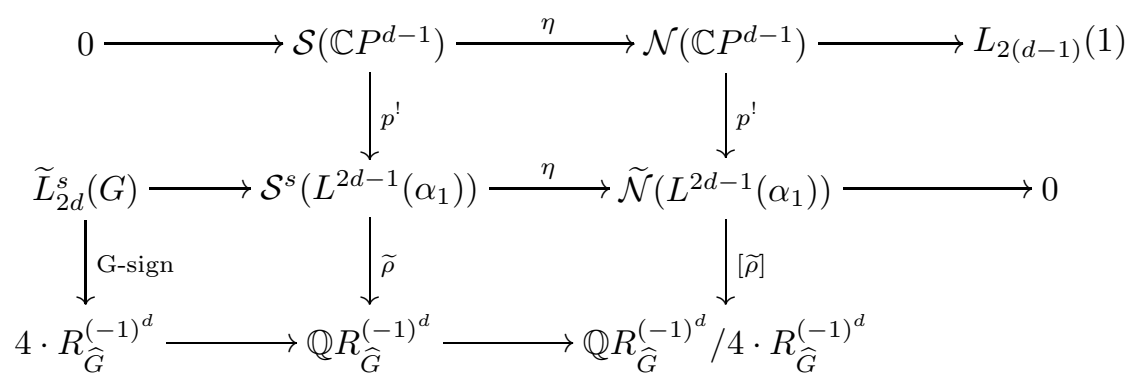

where the maps $p^{!}, \eta$ are homomorphisms. The composition $\widetilde{\rho} \circ p^{!}$is a homomorphism by Wal99, Theorem 14C.4]. Hence also the composition $[\widetilde{\rho}] \circ \eta \circ p^{!}$is a homomorphism.

If $d=2 e$ then $\eta \circ p^{!}$is surjective which implies that $[\widetilde{\rho}]$ is a homomorphism. If $d=2 e+1$ then $\eta \circ p^{!}$is not surjective. But we have the following commutative 
diagram:

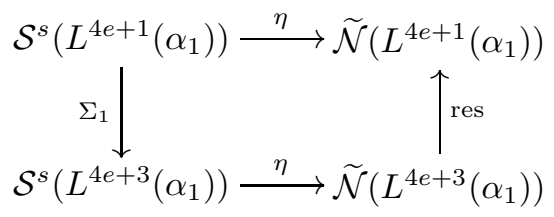

where res is bijective and we have $\widetilde{\rho}\left(\Sigma_{1}(x)\right)=f \cdot \widetilde{\rho}(x)$. Now we can use Lemma 3.6 and calculate:

$$
\begin{aligned}
{[\widetilde{\rho}](\eta(x)+\eta(y)) } & =[\widetilde{\rho}](\eta(x+y))=[\widetilde{\rho}(x+y)]=[g \cdot f \cdot \widetilde{\rho}(x+y)] \\
& =\left[g \cdot \widetilde{\rho}\left(\Sigma_{1}(x+y)\right)\right]=\left[g \cdot \widetilde{\rho}\left(\Sigma_{1}(x)+\Sigma_{1}(y)\right)\right] \\
& =[g] \cdot[\widetilde{\rho}]\left(\eta \Sigma_{1}(x)+\eta \Sigma_{1}(y)\right)=[g] \cdot[\widetilde{\rho}]\left(\eta \Sigma_{1}(x)\right)+[\widetilde{\rho}]\left(\eta \Sigma_{1}(y)\right) \\
& =[g] \cdot\left(\left[\widetilde{\rho}\left(\Sigma_{1}(x)\right)\right]+\left[\widetilde{\rho}\left(\Sigma_{1}(y)\right)\right]\right)=[g] \cdot[f] \cdot([\widetilde{\rho}(x)]+[\widetilde{\rho}(y)]) \\
& =[\widetilde{\rho}](\eta(x))+[\widetilde{\rho}](\eta(y)) .
\end{aligned}
$$

This finishes the proof.

Corollary 3.8. The function $\widetilde{\rho}: \mathcal{S}^{s}\left(L^{2 d-1}\left(\alpha_{1}\right)\right) \longrightarrow \mathbb{Q} R_{\widehat{G}}^{(-1)^{d}}$ is a homomorphism.

Proof. It is enough to show that for every $y, y^{\prime} \in \tilde{\mathcal{N}}\left(L^{2 d-1}\left(\alpha_{1}\right)\right)$ there exist elements (not necessarily unique) $a, a^{\prime}$ in $\mathcal{S}^{s}\left(L^{2 d-1}\left(\alpha_{1}\right)\right)$ such that $\eta(a)=y, \eta\left(a^{\prime}\right)=y^{\prime}$ and $\widetilde{\rho}\left(a+a^{\prime}\right)=\widetilde{\rho}(a)+\widetilde{\rho}\left(a^{\prime}\right)$. If this holds then for any $x, x^{\prime} \in \mathcal{S}^{s}\left(L^{2 d-1}\left(\alpha_{1}\right)\right)$ choose $a$ and $a^{\prime}$ as above corresponding to the classes $\eta(x), \eta\left(x^{\prime}\right) \in \mathcal{N}\left(L^{2 d-1}\left(\alpha_{1}\right)\right)$. Then $x=a+\partial(b)$ and $x^{\prime}=a^{\prime}+\partial\left(b^{\prime}\right)$ for some $b, b^{\prime} \in \widetilde{L}_{2 d}^{s}(G)$ and

$$
\begin{aligned}
\widetilde{\rho}\left(x+x^{\prime}\right) & =\widetilde{\rho}\left(a+\partial(b)+a^{\prime}+\partial\left(b^{\prime}\right)\right)=\widetilde{\rho}\left(a+a^{\prime}\right)+\widetilde{\rho}\left(\partial(b)+\partial\left(b^{\prime}\right)\right) \\
& =\widetilde{\rho}(a)+\widetilde{\rho}\left(a^{\prime}\right)+\widetilde{\rho}(\partial(b))+\widetilde{\rho}\left(\partial\left(b^{\prime}\right)\right)=\widetilde{\rho}(x)+\widetilde{\rho}\left(x^{\prime}\right) .
\end{aligned}
$$

When $d=2 e$ we can associate to a given $y \in \mathcal{N}\left(L^{2 d-1}\left(\alpha_{1}\right)\right)$ an $a \in \mathcal{S}^{s}\left(L^{2 d-1}\left(\alpha_{1}\right)\right)$ coming from the $\mathcal{S}\left(\mathbb{C} P^{d-1}\right)$, i.e. $a=p^{!}(b)$ where $b \in \mathcal{S}\left(\mathbb{C} P^{d-1}\right)$ such that $p^{!}(\eta(b))=$ $y$. When we have $y, y^{\prime} \in \mathcal{N}\left(L^{2 d-1}\left(\alpha_{1}\right)\right)$ then $\widetilde{\rho}\left(a+a^{\prime}\right)=\widetilde{\rho}\left(p^{!}(b)+p^{!}\left(b^{\prime}\right)\right)=$ $\widetilde{\rho}\left(p^{!}\left(b+b^{\prime}\right)\right)=\operatorname{res}\left(\widetilde{\rho}_{S^{1}}\left(b+b^{\prime}\right)\right)=\operatorname{res}\left(\widetilde{\rho}_{S^{1}}(b)+\widetilde{\rho}_{S^{1}}\left(b^{\prime}\right)\right)=\operatorname{res}\left(\widetilde{\rho}_{S^{1}}(b)\right)+\operatorname{res}\left(\widetilde{\rho}_{S^{1}}\left(b^{\prime}\right)\right)=$ $\widetilde{\rho}(a)+\widetilde{\rho}\left(a^{\prime}\right)$. Here res denotes the map on the representation rings induced by the inclusion $G<S^{1}$.

When $d=2 e+1$ it follows from Proposition 3.7 that for $a, a^{\prime} \in \mathcal{S}^{s}\left(L^{2 d-1}\left(\alpha_{1}\right)\right)$ we have $\widetilde{\rho}\left(a+a^{\prime}\right)=\widetilde{\rho}(a)+\widetilde{\rho}\left(a^{\prime}\right)+z$ for some $z \in 4 \cdot R_{\widehat{G}}^{-}$. Our task is to show $z=0$ for any choice of $a, a^{\prime}$. We use the fact that $\Sigma_{1}$ is a homomorphism and that we have already proved the claim for $d=2 e+2$. That implies $\widetilde{\rho}\left(\Sigma_{1}\left(a+a^{\prime}\right)\right)=$ $\widetilde{\rho}\left(\Sigma_{1}(a)+\Sigma_{1}\left(a^{\prime}\right)\right)=\widetilde{\rho}\left(\Sigma_{1}(a)\right)+\widetilde{\rho}\left(\Sigma_{1}\left(a^{\prime}\right)\right)=f \cdot \widetilde{\rho}(a)+f \cdot \widetilde{\rho}\left(a^{\prime}\right)$. On the other hand $\widetilde{\rho}\left(\Sigma_{1}\left(a+a^{\prime}\right)\right)=f \cdot \widetilde{\rho}\left(a+a^{\prime}\right)=f \cdot \widetilde{\rho}(a)+f \cdot \widetilde{\rho}\left(a^{\prime}\right)+f \cdot z$. Hence $f \cdot z=0$ and therefore $z=g \cdot f \cdot z=0$ by Lemma 3.6 .

Now we proceed to the case of $\alpha_{k}$ where $k \in \mathbb{N}$ is such that $(N, k)=1$.

Proposition 3.9. The function $[\widetilde{\rho}]: \mathcal{N}\left(L^{2 d-1}\left(\alpha_{k}\right)\right) \longrightarrow \mathbb{Q} R_{\widehat{G}}^{(-1)^{d}} / 4 \cdot R_{\widehat{G}}^{(-1)^{d}}$ is a homomorphism.

Proof. We will use the result for $\alpha_{1}$ and the homeomorphisms

$$
L^{2 d+1}\left(\alpha_{k}\right) \cong L^{2 d-1}\left(\alpha_{1}\right) * L^{1}\left(\alpha_{k}\right) \quad \text { and } \quad L^{2 d+1}\left(\alpha_{k}\right) \cong L^{2 d-1}\left(\alpha_{k}\right) * L^{1}\left(\alpha_{1}\right)
$$


and formulas (3.2) and (3.3). For $d=2 e$ recall the diagram

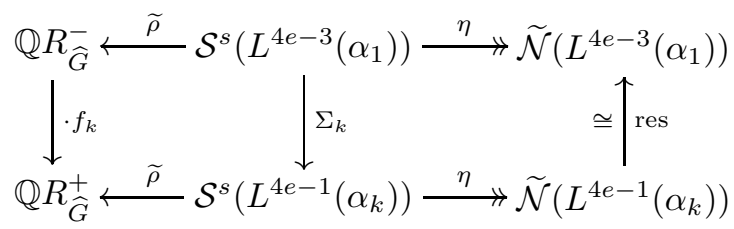

It follows that the composition $[\widetilde{\rho}] \circ(\text { res })^{-1} \circ \eta$ is a homomorphism. Since the composition (res) ${ }^{-1} \circ \eta$ is surjective, we obtain that the function $[\widetilde{\rho}]: \mathcal{N}\left(L^{4 e-1}\left(\alpha_{k}\right)\right) \rightarrow$ $\mathbb{Q} R_{\widehat{G}}^{(-1)^{d}} / 4 \cdot R_{\widehat{G}}^{(-1)^{d}}$ is a homomorphism. For $d=2 e+1$ recall the diagram

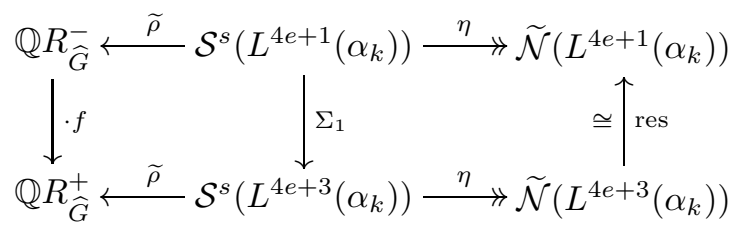

and use a similar reasoning.

Corollary 3.10. The function $\widetilde{\rho}: \mathcal{S}^{s}\left(L^{2 d-1}\left(\alpha_{k}\right)\right) \longrightarrow \mathbb{Q} R_{\widehat{G}}^{(-1)^{d}}$ is a homomorphism.

Proof. Just as in the case $\alpha_{1}$ it is enough to find in each normal cobordism class an element such that the addition works for these representatives. Consider the diagrams (3.7) and (3.8). In the case $d=2 e$ we can choose in each normal cobordism class an element coming from $\mathcal{S}^{s}\left(L^{4 e-3}\left(\alpha_{1}\right)\right)$ and in the case $d=2 e+1$ choose in each normal cobordism class an element coming from $\mathcal{S}^{s}\left(L^{4 e+1}\left(\alpha_{k}\right)\right)$. Then use the fact that we have already proved the proposition in the case $d=2 e+2$ and Lemma 3.6 just as in the case $\alpha_{1}$.

Corollary 3.11. The function $\widetilde{\rho}: \mathcal{S}^{s}\left(L^{2 d-1}(\alpha)\right) \longrightarrow \mathbb{Q} R_{\widehat{G}}^{(-1)^{d}}$ is a homomorphism.

The proof is the same as the proof of [MW08, Corollary 4.16].

\subsection{Formulas.}

In addition to the information we obtained in the previous subsection we need formulas to calculate the homomorphism $[\widetilde{\rho}]$ in some cases. The formulas and the proofs are generalizations of the similar formulas in [MW08, section 4.2]. We note that we will need these formulas only in the case $N=2^{K} \cdot M$ for $K \geq 1$ and hence we can assume $2 \mid N$ in this subsection. The starting point is the following:

Theorem 3.12. Wal99, Theorem 14C.4] Let $a=\left[h: Q \rightarrow \mathbb{C} P^{d-1}\right]$ be an element in $\mathcal{S}^{s}\left(\mathbb{C} P^{d-1}\right)$. Then for $t \in S^{1}$

$$
\widetilde{\rho}_{S^{1}}(t, a)=\sum_{1 \leq i \leq\lfloor d / 2\rfloor-1} 8 \cdot \mathbf{s}_{4 i}(\eta(a)) \cdot\left(f^{d-2 i}-f^{d-2 i-2}\right) \in \mathbb{C},
$$

where $f=(1+t) /(1-t)$.

This was used to obtain a formula for $\widetilde{\rho}$ for fake lens spaces which fiber over the fake complex projective spaces in [Wal99, Theorem 14E.8]. From that we obtain a formula for $[\widetilde{\rho}]$ when $d=2 e$ since in that case there exists in each normal cobordism class of fake lens spaces a fake lens space which fibers over a fake complex projective space. When $d=2 e+1$ we need to adapt the trick from MW08, Lemma 4.10, $4.11]$ to our situation. This is done as follows. 
Lemma 3.13. Let $d=2 e+1$ and let $c \in \mathcal{S}^{s}\left(L^{2 d-1}\left(\alpha_{1}\right)\right)$ be such that $c=b+a$ where $b=p^{!}(\widetilde{b})$ for some $\widetilde{b} \in \mathcal{S}\left(\mathbb{C} P^{d-1}\right)$ with $s_{2 i}=\mathbf{s}_{2 i}(\eta \widetilde{b})$ and a is such that there exists an $\widetilde{a} \in \mathcal{N}\left(\mathbb{C} P^{d-1}\right)$ such that $\mathbf{s}_{2 i}(\widetilde{a})=0$ for $i<2 e, s_{4 e}=\mathbf{s}_{4 e}(\widetilde{a})$ and $\eta(a)=p^{!}(\widetilde{a})$. Then

$$
\tilde{\rho}(c)=\sum_{1 \leq i \leq\lfloor d / 2\rfloor-1} 8 \cdot s_{4 i} \cdot\left(f^{d-2 i}-f^{d-2 i-2}\right)+8 \cdot s_{4 e} \cdot f+z \quad \in \quad \mathbb{Q} R_{\widehat{G}}^{-}
$$

for some $z \in 4 \cdot R_{\widehat{G}}^{-}$.

Proof. We will show that, if $s_{4 i}=0$ for all $i<e$ and $s_{4 e}=1$ then

$$
\widetilde{\rho}(a)=8 f+z \quad \in \quad \mathbb{Q} R_{\widehat{G}}^{-}
$$

for some $z \in 4 \cdot R_{\widehat{G}}^{-}$. The proof of the general case is the same, but the formulas are more complicated.

We will use the suspension map $\Sigma_{1}$. Our assumptions mean that $\eta(a)$ is not in the image of the composition $\mathcal{S}\left(\mathbb{C} P^{d-1}\right) \rightarrow \mathcal{N}\left(\mathbb{C} P^{d-1}\right) \rightarrow \mathcal{N}\left(L^{2 d-1}\left(\alpha_{1}\right)\right)$. However, diagram (2.8) and the formula from Theorem 3.12 tells us that $\eta\left(\Sigma_{1}(a)\right)$ is in the image of $\mathcal{S}\left(\mathbb{C} P^{(d+1)-1}\right) \rightarrow \mathcal{N}\left(\mathbb{C} P^{(d+1)-1}\right) \rightarrow \mathcal{N}\left(L^{2(d+1)-1}\left(\alpha_{1}\right)\right)$ and we have

$$
f \cdot \widetilde{\rho}(a)+y=8 \cdot 1 \cdot\left(f^{2}-1\right) \in \mathbb{Q} R_{\widehat{G}}^{+}
$$

for some $y \in 4 \cdot R_{\widehat{G}}^{+}$. We obtain the desired identity by the following calculation. Let $\widehat{\rho} \in \mathbb{Q}[\chi]$ and $\widehat{y} \in 4 \cdot \mathbb{Z}[\chi]$ be representatives for $\widetilde{\rho}(a)$ and $y$. Then

$$
\begin{array}{rlrl}
(1+\chi)(1-\chi) \widehat{\rho}+(1-\chi)^{2} \widehat{y} & \equiv 8 \cdot(4 \chi) & \bmod \left\langle 1+\chi+\cdots+\chi^{N-1}\right\rangle \\
(1+\chi)(1-\chi) \widehat{\rho}+(1-\chi)^{2}(\widehat{y}+8) & \equiv 8 \cdot(1+\chi)^{2} \quad \bmod \left\langle 1+\chi+\cdots+\chi^{N-1}\right\rangle \\
(1+\chi)(1-\chi) \widehat{\rho}+(1-\chi)^{2}(\widehat{y}+8) & =8 \cdot(1+\chi)^{2}+g(\chi)\left(1+\chi+\cdots+\chi^{N-1}\right) \in \mathbb{Q}[\chi]
\end{array}
$$

for some $g(\chi) \in \mathbb{Q}[\chi]$. Because 2 divides $N$ we get $\widehat{y}+8=(1+\chi) \cdot w(\chi)$ for some $w(\chi) \in \mathbb{Q}[\chi]$. Since $\widehat{y}+8 \in 4 \cdot \mathbb{Z}[\chi]$, we obtain $w(\chi) \in 4 \cdot \mathbb{Z}[\chi]$. Further write $g(\chi)=2 r+(1+\chi) g^{\prime}(\chi)=r(1-\chi)+(1+\chi)\left(r+g^{\prime}(\chi)\right)$ for $r \in \mathbb{Q}, g^{\prime}(\chi) \in \mathbb{Q}[\chi]$. We have

$$
(1-\chi) \cdot \widehat{\rho}+(1-\chi)^{2} \cdot w(\chi)=8 \cdot(1+\chi)+g(\chi) \cdot\left(1+\chi^{2}+\cdots \chi^{N-2}\right) \in \mathbb{Q}[\chi]
$$

and further modulo $\left\langle 1+\chi+\cdots+\chi^{N-1}\right\rangle$

$$
\begin{gathered}
(1-\chi) \cdot \widehat{\rho}+(1-\chi)^{2} \cdot w(\chi) \equiv 8 \cdot(1+\chi)+r \cdot(1-\chi) \cdot\left(1+\chi^{2}+\cdots \chi^{N-2}\right) \\
\widehat{\rho}+(1-\chi) \cdot w(\chi) \equiv 8 \cdot f+r \cdot\left(1+\chi^{2}+\cdots \chi^{N-2}\right)
\end{gathered}
$$

Now $(1-\chi) \cdot w(\chi)=(2-(1+\chi)) \cdot w(\chi)=2 \cdot w(\chi)-(\widehat{y}+8)$. Further $2 \cdot w(\chi)=$ $w^{+}(\chi)+w^{-}(\chi)$, where $w^{ \pm}(\chi) \in 4 \cdot R_{\widehat{G}}^{( \pm 1)}$. Hence

$$
\widetilde{\rho}(a)-8 \cdot f+w^{-}(\chi)=(\widehat{y}+8)-w^{+}(\chi)+r \cdot\left(1+\chi^{2}+\cdots+\chi^{N-2}\right)
$$

in $\mathbb{Q}[\chi] /\left\langle 1+\chi+\cdots+\chi^{N-1}\right\rangle$, while the left hand side of the equation lies in the $(-1)$-eigenspace and the right-hand side lies in the $(+1)$-eigenspace and hence both are equal to 0 . It follows that

$$
\widetilde{\rho}(a)=8 \cdot f-w^{-}(\chi) .
$$

Putting $z=-w^{-}(\chi)$ yields is the desired formula.

Proposition 3.14. Let $t=\left(t_{2 i}\right)_{i} \in \widetilde{\mathcal{N}}\left(L^{2 d-1}\left(\alpha_{1}\right)\right)_{(2)}$ and $\bar{t}_{4 i} \in \mathbb{Z}(1 \leq i \leq c)$ with $\bar{t}_{4 i} \equiv t_{4 i} \bmod 2^{K}$ and $\bar{t}_{4 i} \equiv 0 \bmod M^{c}$. Then we have for the homomorphism 


$$
\begin{aligned}
{[\widetilde{\rho}]: \widetilde{\mathcal{N}}\left(L^{2 d-1}\left(\alpha_{1}\right)\right)_{(2)} } & \longrightarrow \mathbb{Q} R_{\widehat{G}}^{(-1)^{d}} / 4 \cdot R_{\widehat{G}}^{(-1)^{d}} \text { that } \\
d=2 e & :[\widetilde{\rho}](t)=\sum_{i=1}^{e-1} 8 \cdot \bar{t}_{4 i} \cdot f^{d-2 i-2} \cdot\left(f^{2}-1\right) \\
d=2 e+1 & :[\widetilde{\rho}](t)=\sum_{i=1}^{e-1} 8 \cdot \bar{t}_{4 i} \cdot f^{d-2 i-2} \cdot\left(f^{2}-1\right)+8 \cdot \bar{t}_{4 e} \cdot f .
\end{aligned}
$$

Proof. It is enough to find for each $t \in \widetilde{\mathcal{N}}\left(L^{2 d-1}\left(\alpha_{1}\right)\right)_{(2)}$ some $a \in \mathcal{S}^{s}\left(L^{2 d-1}\left(\alpha_{1}\right)\right)$ with $\mathbf{t}(\eta(a))=(t, 0)$ and for which we can calculate $\widetilde{\rho}(a)$. If $d=2 e$ then there is for each normal cobordism class a fake lens space which fibers over a fake complex projective space and Theorem 3.12 gives the desired formula. If $d=2 e+1$ we apply instead Lemma 3.13 to get the desired formula.

Proposition 3.15. Let $t=\left(t_{2 i}\right)_{i} \in \tilde{\mathcal{N}}\left(L^{2 d-1}\left(\alpha_{k}\right)\right)_{(2)}$ and $\bar{t}_{4 i} \in \mathbb{Z}(1 \leq i \leq c)$ with $\bar{t}_{4 i} \equiv t_{4 i} \bmod 2^{K}$ and $\bar{t}_{4 i} \equiv 0 \bmod M^{c}$. Then we have for the homomorphism $[\widetilde{\rho}]: \widetilde{\mathcal{N}}\left(L^{2 d-1}\left(\alpha_{k}\right)\right)_{(2)} \longrightarrow \mathbb{Q} R_{\widehat{G}}^{(-1)^{d}} / 4 \cdot R_{\widehat{G}}^{(-1)^{d}}$ that

$$
\begin{aligned}
d=2 e:[\widetilde{\rho}](t) & =\sum_{i=1}^{e-1} 8 \cdot \bar{t}_{4 i} \cdot f_{k}^{\prime} \cdot f^{d-2 i-2} \cdot\left(f^{2}-1\right) \\
d=2 e+1:[\widetilde{\rho}](t) & =\sum_{i=1}^{e-1} 8 \cdot \bar{t}_{4 i} \cdot f_{k}^{\prime} \cdot f^{d-2 i-2} \cdot\left(f^{2}-1\right)+8 \cdot \bar{t}_{4 e} \cdot f_{k}^{\prime} \cdot f .
\end{aligned}
$$

Proof. We will use the calculation for $\alpha_{1}$ and the homeomorphisms

$$
L^{2 d+1}\left(\alpha_{k}\right) \cong L^{2 d-1}\left(\alpha_{1}\right) * L^{1}\left(\alpha_{k}\right) \quad \text { and } \quad L^{2 d+1}\left(\alpha_{k}\right) \cong L^{2 d-1}\left(\alpha_{k}\right) * L^{1}\left(\alpha_{1}\right)
$$

For $d=2 e$ recall the diagram

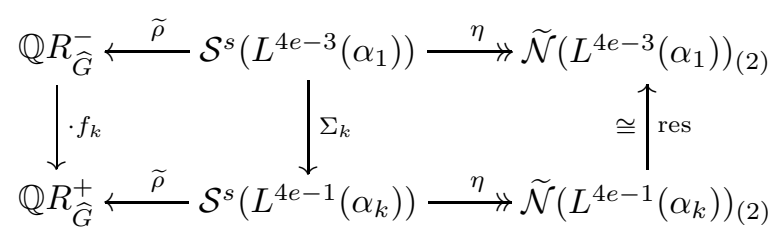

Let $t \in \mathcal{N}\left(L^{4 e-1}\left(\alpha_{k}\right)\right)$. Choose $x \in \mathcal{S}^{s}\left(L^{4 e-3}\left(\alpha_{1}\right)\right)$ such that $\mathbf{t}(\eta(x))=t=\operatorname{res}(t)$. Then we have $\mathbf{t}\left(\eta\left(\Sigma_{k}(x)\right)\right)=t$ and $[\widetilde{\rho}]\left(\eta\left(\Sigma_{k}(x)\right)\right)=\left[\widetilde{\rho}(x) \cdot f_{k}\right]$ can be calculated using the formulas from the case $k=1$.

For $d=2 e+1$ recall the diagram

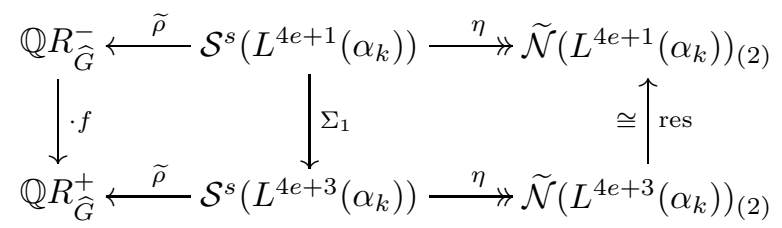

Let $t \in \tilde{\mathcal{N}}\left(L^{4 e+1}\left(\alpha_{k}\right)\right)$. Choose $x \in \mathcal{S}^{s}\left(L^{4 e+1}\left(\alpha_{1}\right)\right)$ such that $\mathbf{t}(\eta(x))=t$. Then we have $\mathbf{t}\left(\eta\left(\Sigma_{1}(x)\right)\right)=t$ and $\widetilde{\rho}\left(\Sigma_{1}(x)\right)=\widetilde{\rho}(x) \cdot f$. We obtain the equation

$$
f \cdot \widetilde{\rho}(x)+y=\sum_{i=1}^{e-1} 8 \cdot \bar{t}_{4 i} \cdot f_{k}^{\prime} \cdot f^{d+1-2 i-2} \cdot\left(f^{2}-1\right)+8 \cdot \bar{t}_{4 e} \cdot f_{k}^{\prime} \cdot\left(f^{2}-1\right) \in \mathbb{Q} R_{\widetilde{G}}^{+}
$$

for some $y \in 4 \cdot R_{\widehat{G}}^{+}$using the formulas from the case $d=2 e+2$ which we have already dealt with. Now a modification of the argument from the proof of Lemma 3.13 can be used to obtain the formula for $[\widetilde{\rho}](\eta(x))$. 


\section{REVISION}

In this section we show how to use Proposition 3.5 to calculate the structure set of the fake lens spaces. We also remind the reader the calculations when $N=M$ odd and $N=2^{K}$ which are used below to solve the general case.

Proposition 4.1. Let $d \geq 3$ and $N=2^{K} \cdot M$ with $K \geq 1, M$ odd, and $G=\mathbb{Z}_{N}$. Then we have

$$
\mathcal{S}^{s}\left(L_{N}^{2 d-1}(\alpha)\right) \cong \bar{\Sigma}_{N}(d) \oplus \operatorname{ker}\left[\widetilde{\rho}_{N}\right]
$$

with $\bar{\Sigma}_{N}(d):=\operatorname{im}\left(\widetilde{\rho}_{N}: \mathcal{S}^{s}(L(\alpha)) \rightarrow \mathbb{Q} R_{\widehat{G}}^{(-1)^{d}}\right)$. The rank of the free abelian group $\bar{\Sigma}_{N}(d)$ equals the rank of $\widetilde{L}_{2 d}^{s}(G)$.

The proof is the same as the proof of Theorem 5.1 in [MW08. So in order to calculate the structure set one needs to calculate the kernel $\operatorname{ker}\left[\widetilde{\rho}_{N}\right]$. This is essentially what Wall has done in Wal99, chapter 14E] and what we have done in MW08 in the special cases.

Theorem 4.2. (Wal99, chapter 14E]) Let $d \geq 3$. If $N=M$ is odd then we have

$$
\mathcal{S}^{s}\left(L_{M}^{2 d-1}(\alpha)\right) \cong \bar{\Sigma}_{M}(d) \text {. }
$$

Equivalently, $\operatorname{ker}\left[\widetilde{\rho}_{M}\right]=0$.

Theorem 4.3. ([MW08, Theorem 1.2]) Let $d \geq 3$. If $N=2^{K}$ with $K \geq 0$ then we have

$$
\mathcal{S}^{s}\left(L_{2^{K}}^{2 d-1}(\alpha)\right) \cong \bar{\Sigma}_{2^{K}}(d) \oplus \operatorname{ker}\left[\widetilde{\rho}_{2^{K}}\right] \cong \bar{\Sigma}_{2^{K}}(d) \oplus \bigoplus_{i=1}^{c} \mathbb{Z}_{2^{\min \{K, 1\}}} \oplus \bigoplus_{i=1}^{c} \mathbb{Z}_{2^{\min \{K, 2 i\}}}
$$

where $\bar{\Sigma}_{2^{K}}(d)$ is a free abelian group of rank $N / 2-1$ if $d=2 e+1$ and $N / 2$ if $d=2 e$ and $c=\lfloor(d-1) / 2\rfloor$.

\section{Calculations}

In this section we give the proof of Theorem 1.2 by calculating $\operatorname{ker}\left[\widetilde{\rho}_{N}\right]$. Notice that for any fake lens space $L_{N}^{2 d-1}(\alpha)$ there exists $k \in \mathbb{N}$ and a homotopy equivalence $h: L_{N}^{2 d-1}(\alpha) \longrightarrow L_{N}^{2 d-1}\left(\alpha_{k}\right)$ (see for instance [MW08, Corollary 2.4]). It induces an isomorphism $h_{*}: \mathcal{S}^{s}\left(L_{N}^{2 d-1}(\alpha)\right) \rightarrow \mathcal{S}^{s}\left(L_{N}^{2 d-1}\left(\alpha_{k}\right)\right)$. Hence it suffices to consider the case $\alpha=\alpha_{k}$. In Proposition 5.1 we calculate

$$
\operatorname{ker}\left[\widetilde{\rho}_{N}\right]: \widetilde{\mathcal{N}}\left(L_{N}^{2 d-1}\left(\alpha_{k}\right)\right) \rightarrow \mathbb{Q} R_{\widehat{\mathbb{Z}}_{N}}^{(-1)^{d}} / 4 \cdot R_{\widehat{\mathbb{Z}}_{N}}^{(-1)^{d}} .
$$

Theorem 1.2 follows from this proposition together with Proposition 4.1 and Theorems $4.2,4.3$

We need a little preparation for Proposition 5.1. Notice that we have commutative diagrams

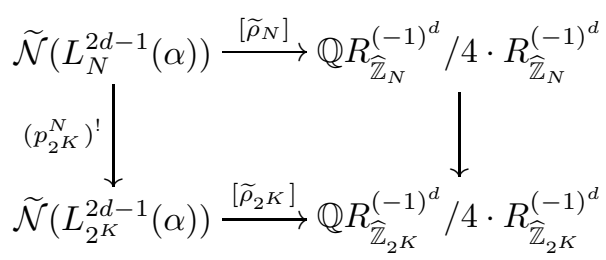


and

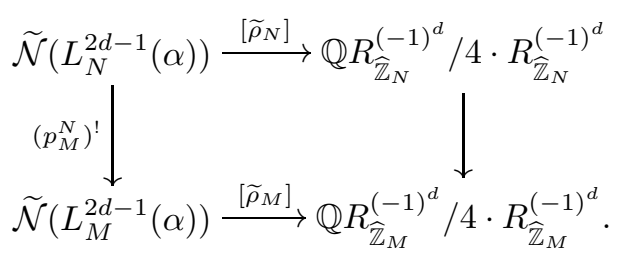

This follows from the fact that the $\widetilde{\rho}$-invariant is natural with respect to the restriction maps. Note also that if we use our identification of the right hand terms with the polynomial rings, then the right hand vertical maps are given by $\chi \mapsto \chi$, see Remark 3.4. We also have an isomorphism

$$
\tilde{\mathcal{N}}\left(L_{N}\right) \cong \widetilde{\mathcal{N}}\left(L_{N}\right)_{(2)} \oplus \widetilde{\mathcal{N}}\left(L_{N}\right)_{(o d d)} \stackrel{\left(p_{2 K}^{N}\right)^{!} \oplus\left(p_{M}^{N}\right)^{!}}{\longrightarrow} \widetilde{\mathcal{N}}\left(L_{2^{K}}\right) \oplus \widetilde{\mathcal{N}}\left(L_{M}\right)
$$

where $L_{?}=L_{?}^{2 d-1}(\alpha)$, see (2.7).

Proposition 5.1. Let $N=2^{K} \cdot M$ with $K \geq 1, M>1$ odd and let $d \geq 3$. Then we have

$$
\begin{aligned}
\operatorname{ker}\left[\widetilde{\rho}_{N}\right] & \cong\left(\left(p_{2^{K}}^{N}\right)^{!}\right)^{-1} \operatorname{ker}\left[\widetilde{\rho}_{2^{K}}\right] \oplus\left(\left(p_{M}^{N}\right)^{!}\right)^{-1} \operatorname{ker}\left[\widetilde{\rho}_{M}\right]=\left(\left(p_{2^{K}}^{N}\right)^{!}\right)^{-1} \operatorname{ker}\left[\widetilde{\rho}_{2^{K}}\right] \oplus 0 \\
& \subseteq \widetilde{\mathcal{N}}\left(L_{N}^{2 d-1}\left(\alpha_{k}\right)\right)_{(2)} \oplus \widetilde{\mathcal{N}}\left(L_{N}^{2 d-1}\left(\alpha_{k}\right)\right)_{(\text {odd })} .
\end{aligned}
$$

For the proof of this proposition we need the following two lemmas.

Lemma 5.2. Let $a \in \mathbb{Q}[\chi]$ such that there exist $b, c \in 4 \cdot \mathbb{Z}[\chi]$ satisfying

(1) $a \equiv b \quad \bmod 1+\chi+\cdots+\chi^{2^{K}-1} \quad$ and

(2) $a \equiv c \quad \bmod 1+\chi^{2^{K}}+\cdots+\chi^{2^{K} \cdot(M-1)}$.

Then there exists $d \in 4 \cdot \mathbb{Z}[\chi]$ such that

$$
M \cdot a \equiv d \bmod 1+\chi+\cdots+\chi^{N-1}
$$

holds.

Proof. Our candidate for $d$ is

$$
d:=c \cdot M+(b-c) \cdot\left(1+\chi^{2^{K}}+\cdots+\chi^{2^{K} \cdot(M-1)}\right) .
$$

We have to check that $M \cdot a \equiv d \bmod 1+\chi+\cdots+\chi^{N-1}$ holds. There is the factorization

$$
1+\chi+\cdots+\chi^{N-1}=\left(1+\chi+\cdots+\chi^{2^{K}-1}\right) \cdot\left(1+\chi^{2^{K}}+\cdots+\chi^{2^{K} \cdot(M-1)}\right) .
$$

Notice that the two factors are prime to each other in $\mathbb{Q}[\chi]$ because of

$$
\sum_{i=0}^{M-1}\left(\chi^{2^{K}}\right)^{i} \equiv \sum_{i=0}^{M-1} 1^{i} \equiv M \quad \bmod 1+\chi+\cdots+\chi^{2^{K}-1} .
$$

Therefore, it suffices to check that

$$
\begin{aligned}
& M \cdot a \equiv d \bmod 1+\chi+\cdots+\chi^{2^{K}-1} \quad \text { and } \\
& M \cdot a \equiv d \bmod 1+\chi^{2^{K}}+\cdots+\chi^{2^{K} \cdot(M-1)}
\end{aligned}
$$

hold. But this is true because of equation 5.3 .

Lemma 5.3. Let $k \in \mathbb{N}$ with $(k, N)=1$ and $q \in \mathbb{Z}[x]$. Then

$$
\begin{gathered}
8 \cdot f_{k}^{\prime} \cdot\left(f^{2}-1\right) \cdot M^{2+2 \operatorname{deg}(q)} \cdot q\left(f^{2}\right) \in 4 \cdot \mathbb{Z}[\chi] /\left\langle 1+\chi^{2^{K}}+\cdots+\chi^{2^{K} \cdot(M-1)}\right\rangle \text { and } \\
8 \cdot f_{k}^{\prime} \cdot f \cdot M^{1+2 \operatorname{deg}(q)} \cdot q\left(f^{2}\right) \in 4 \cdot \mathbb{Z}[\chi] /\left\langle 1+\chi^{2^{K}}+\cdots+\chi^{2^{K} \cdot(M-1)}\right\rangle .
\end{gathered}
$$


Proof. Because of the equation

$$
\begin{aligned}
& (1-\chi) \cdot\left(1+\chi+\cdots+\chi^{2^{K}-1}\right) \cdot\left(1+2 \cdot \chi^{2^{K}}+3 \cdot \chi^{2 \cdot 2^{K}}+\cdots+M \cdot \chi^{(M-1) \cdot 2^{K}}\right)= \\
& \left(1+\chi^{2^{K}}+\cdots+\chi^{(M-1) \cdot 2^{K}}\right)-M \cdot \chi^{N}
\end{aligned}
$$

we have in $\mathbb{Q}[\chi] /\left\langle 1+\chi^{2^{K}}+\cdots+\chi^{2^{K} \cdot(M-1)}\right\rangle$ :

$M \cdot f=-(1+\chi) \cdot\left(1+\chi+\cdots+\chi^{2^{K}-1}\right) \cdot\left(1+2 \chi^{2^{K}}+3 \chi^{2 \cdot 2^{K}}+\cdots+M \chi^{(M-1) \cdot 2^{K}}\right)$.

Notice that $f_{k}^{\prime} \in \mathbb{Z}[\chi] /\left\langle 1+\chi^{2^{K}}+\cdots+\chi^{2^{K} \cdot(M-1)}\right\rangle$ because of $f_{k}^{\prime} \in R_{\widehat{\mathbb{Z}}_{N}}$. Therefore, we obtain

$$
\begin{aligned}
8 \cdot f_{k}^{\prime} \cdot\left(f^{2}-1\right) \cdot M^{2+2 \operatorname{deg}(q)} \cdot q\left(f^{2}\right) & \in 4 \cdot \mathbb{Z}[\chi] /\left\langle 1+\chi^{2^{K}}+\cdots+\chi^{2^{K} \cdot(M-1)}\right\rangle \text { and } \\
8 \cdot f_{k}^{\prime} \cdot f \cdot M^{1+2 \operatorname{deg}(q)} \cdot q\left(f^{2}\right) & \in 4 \cdot \mathbb{Z}[\chi] /\left\langle 1+\chi^{2^{K}}+\cdots+\chi^{2^{K} \cdot(M-1)}\right\rangle .
\end{aligned}
$$

Proof of Proposition 5.1. In the sequel we use the identification

$$
\tilde{\mathcal{N}}\left(L_{N}^{2 d-1}\left(\alpha_{k}\right)\right)=\tilde{\mathcal{N}}\left(L_{N}^{2 d-1}\left(\alpha_{k}\right)\right)_{(2)} \oplus \tilde{\mathcal{N}}\left(L_{N}^{2 d-1}\left(\alpha_{k}\right)\right)_{(o d d)} .
$$

Obviously, we have

$$
\operatorname{ker}\left[\widetilde{\rho}_{N}\right] \supseteq \operatorname{ker}\left(\left.\left[\widetilde{\rho}_{N}\right]\right|_{\widetilde{\mathcal{N}}\left(L_{N}^{2 d-1}\left(\alpha_{k}\right)\right)_{(2)}}\right) \oplus \operatorname{ker}\left(\left.\left[\widetilde{\rho}_{N}\right]\right|_{\left.\widetilde{\mathcal{N}}\left(L_{N}^{2 d-1}\left(\alpha_{k}\right)\right)_{(o d d)}\right)}\right) .
$$

We want to prove the other inclusion as well. Let $x=x_{(2)}+x_{(\text {odd })} \in \operatorname{ker}\left[\widetilde{\rho}_{N}\right]$ with $x_{(2)} \in \tilde{\mathcal{N}}\left(L_{N}^{2 d-1}\left(\alpha_{k}\right)\right)_{(2)}$ and $x_{(o d d)} \in \tilde{\mathcal{N}}\left(L_{N}^{2 d-1}\left(\alpha_{k}\right)\right)_{(o d d)}$. Notice that $2^{K} \cdot x_{(2)}=0$ and $M^{c} \cdot x_{(o d d)}=0$ hold. Since $M$ is odd, there exist $a, b \in \mathbb{Z}$ such that $a \cdot 2^{K}+b$. $M^{c}=1$. We conclude

$$
\left[\widetilde{\rho}_{N}\right]\left(x_{(2)}\right)=\left[\widetilde{\rho}_{N}\right]\left(x_{(2)}-a \cdot 2^{K} \cdot x_{(2)}\right)=\left[\widetilde{\rho}_{N}\right]\left(b \cdot M^{c} \cdot x_{(2)}\right)=\left[\widetilde{\rho}_{N}\right]\left(b \cdot M^{c} \cdot x\right)=0
$$

and

$\left[\widetilde{\rho}_{N}\right]\left(x_{(\text {odd })}\right)=\left[\widetilde{\rho}_{N}\right]\left(x_{(\text {odd })}-b \cdot M^{c} \cdot x_{(\text {odd })}\right)=\left[\widetilde{\rho}_{N}\right]\left(a \cdot 2^{K} \cdot x_{(\text {odd })}\right)=\left[\widetilde{\rho}_{N}\right]\left(a \cdot 2^{K} \cdot x\right)=0$.

This shows

$$
\operatorname{ker}\left[\widetilde{\rho}_{N}\right]=\operatorname{ker}\left(\left.\left[\widetilde{\rho}_{N}\right]\right|_{\widetilde{\mathcal{N}}\left(L_{N}^{2 d-1}\left(\alpha_{k}\right)\right)_{(2)}}\right) \oplus \operatorname{ker}\left(\left.\left[\widetilde{\rho}_{N}\right]\right|_{\left.\widetilde{\mathcal{N}}\left(L_{N}^{2 d-1}\left(\alpha_{k}\right)\right)_{(o d d)}\right) .}\right.
$$

It remains to prove that we have $\operatorname{ker}\left(\left.\left[\widetilde{\rho}_{N}\right]\right|_{\widetilde{\mathcal{N}}\left(L_{N}^{2 d-1}\left(\alpha_{k}\right)\right)_{(2)}}\right)=\left(\left(p_{2^{K}}^{N}\right)^{!}\right)^{-1} \operatorname{ker}\left[\widetilde{\rho}_{2^{K}}\right]$ and $\operatorname{ker}\left(\left.\left[\widetilde{\rho}_{N}\right]\right|_{\widetilde{\mathcal{N}}\left(L_{N}^{2 d-1}\left(\alpha_{k}\right)\right)_{(o d d)}}\right)=\left(\left(p_{M}^{N}\right)^{!}\right)^{-1} \operatorname{ker}\left[\widetilde{\rho}_{M}\right]=0$. From diagram 5.2 we conclude $\operatorname{ker}\left(\left.\left[\widetilde{\rho}_{N}\right]\right|_{\widetilde{\mathcal{N}}\left(L_{N}^{2 d-1}\left(\alpha_{k}\right)\right)_{(\text {odd })}}\right) \subseteq\left(\left(p_{M}^{N}\right)^{!}\right)^{-1} \operatorname{ker}\left[\widetilde{\rho}_{M}\right]$. Proposition 3.5 implies that $\eta: \mathcal{S}^{s}\left(L_{M}^{2 d-1}\left(\alpha_{k}\right)\right) \rightarrow \widetilde{\mathcal{N}}\left(L_{M}^{2 d-1}\left(\alpha_{k}\right)\right)$ induces an isomorphism $\operatorname{ker}\left(\widetilde{\rho}_{M}\right) \cong \operatorname{ker}\left[\widetilde{\rho}_{M}\right]$. Notice that $\operatorname{ker}\left[\widetilde{\rho}_{M}\right]=0$ by Theorem 4.2. Hence we obtain

$$
\left.\operatorname{ker}\left(\left.\left[\widetilde{\rho}_{N}\right]\right|_{\widetilde{\mathcal{N}}\left(L_{N}^{2 d-1}\left(\alpha_{k}\right)\right)_{(o d d)}}\right)\right)=\left(\left(p_{M}^{N}\right)^{!}\right)^{-1} \operatorname{ker}\left[\widetilde{\rho}_{M}\right]=0
$$

Diagram 5.1 implies

$$
\operatorname{ker}\left(\left.\left[\widetilde{\rho}_{N}\right]\right|_{\widetilde{\mathcal{N}}\left(L_{N}^{2 d-1}\left(\alpha_{k}\right)\right)_{(2)}}\right) \subseteq\left(\left(p_{2^{K}}^{N}\right)^{!}\right)^{-1} \operatorname{ker}\left[\widetilde{\rho}_{2^{K}}\right] .
$$

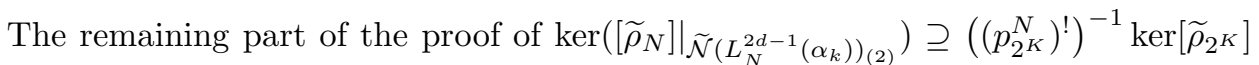
needs more effort. Let $x_{(2)} \in\left(\left(p_{2^{K}}^{N}\right)^{!}\right)^{-1} \operatorname{ker}\left[\widetilde{\rho}_{2^{K}}\right] \subseteq \tilde{\mathcal{N}}\left(L_{N}^{2 d-1}\left(\alpha_{k}\right)\right)_{(2)}$. Recall from 
(2.7) that we have isomorphisms

$$
\begin{aligned}
\mathbf{t}_{(2)} & =\left(\mathbf{t}_{2 i}\right)_{i}: \tilde{\mathcal{N}}\left(L_{N}^{2 d-1}\left(\alpha_{k}\right)\right)_{(2)} \cong \bigoplus_{i=1}^{c} \mathbb{Z}_{2^{K}} \oplus \bigoplus_{i=1}^{c} \mathbb{Z}_{2} \\
\mathbf{t} & =\left(\mathbf{t}_{2 i}\right)_{i}: \tilde{\mathcal{N}}\left(L_{2^{K}}^{2 d-1}\left(\alpha_{k}\right)\right) \cong \bigoplus_{i=1}^{c} \mathbb{Z}_{2^{K}} \oplus \bigoplus_{i=1}^{c} \mathbb{Z}_{2}
\end{aligned}
$$

which commute with the isomorphism $\left(p_{2^{K}}^{N}\right)^{!}$. Choose $\bar{t}_{4 i} \in \mathbb{Z}(1 \leq i \leq c)$ with $\bar{t}_{4 i} \equiv \mathbf{t}_{4 i}\left(\left(p_{2^{K}}^{N}\right)^{!}\left(x_{(2)}\right)\right) \bmod 2^{K}$. From Proposition 3.15 we conclude

$$
\left[\widetilde{\rho}_{2^{K}}\right]\left(\left(p_{2^{K}}^{N}\right)^{!}\left(x_{(2)}\right)\right)= \begin{cases}8 \cdot f_{k}^{\prime} \cdot\left(f^{2}-1\right) \cdot q_{\bar{t}}\left(f^{2}\right) & d \text { even } \\ 8 \cdot f_{k}^{\prime} \cdot f \cdot q_{\bar{t}}\left(f^{2}\right) & d \text { odd }\end{cases}
$$

where the polynomial $q_{\bar{t}} \in \mathbb{Z}[x]$ is defined by

$$
q_{\bar{t}}(x):= \begin{cases}\sum_{i=0}^{c-1} \bar{t}_{4(i+1)} \cdot x^{c-i-1} & d \text { even }, \\ \sum_{i=1}^{c-1} \bar{t}_{4 i} \cdot x^{c-i-1} \cdot(x-1)+\bar{t}_{4 c} & d \text { odd } .\end{cases}
$$

Since $x_{(2)} \in \operatorname{ker}\left[\widetilde{\rho}_{2^{K}}\right]$, we have

$$
\begin{aligned}
8 \cdot f_{k}^{\prime} \cdot\left(f^{2}-1\right) \cdot q_{\bar{t}}\left(f^{2}\right) & \in 4 \cdot R_{\widehat{\mathbb{Z}}_{2 K}} \cong 4 \cdot \mathbb{Z}[\chi] /\left\langle 1+\chi+\cdots+\chi^{2^{K}-1}\right\rangle \quad(d \text { even }), \\
8 \cdot f_{k}^{\prime} \cdot f \cdot q_{\bar{t}}\left(f^{2}\right) & \in 4 \cdot R_{\widehat{\mathbb{Z}}_{2}} \cong 4 \cdot \mathbb{Z}[\chi] /\left\langle 1+\chi+\cdots+\chi^{2^{K}-1}\right\rangle \quad(d \text { odd }) .
\end{aligned}
$$

Lemma 5.3 tells us in respective cases that

$$
\begin{aligned}
8 \cdot f_{k}^{\prime} \cdot\left(f^{2}-1\right) \cdot M^{2+2 \operatorname{deg}\left(q_{\bar{t}}\right)} \cdot q_{\bar{t}}\left(f^{2}\right) & \in 4 \cdot \mathbb{Z}[\chi] /\left\langle 1+\chi^{2^{K}}+\cdots+\chi^{2^{K} \cdot(M-1)}\right\rangle \\
8 \cdot f_{k}^{\prime} \cdot f \cdot M^{1+2 \operatorname{deg}\left(q_{\bar{t}}\right)} \cdot q_{\bar{t}}\left(f^{2}\right) & \in 4 \cdot \mathbb{Z}[\chi] /\left\langle 1+\chi^{2^{K}}+\cdots+\chi^{2^{K} \cdot(M-1)}\right\rangle .
\end{aligned}
$$

Using Lemma 5.2 we obtain in respective cases

$8 \cdot f_{k}^{\prime} \cdot\left(f^{2}-1\right) \cdot M^{3+2 \operatorname{deg}\left(q_{\bar{t}}\right)} \cdot q_{\bar{t}}\left(f^{2}\right) \in 4 \cdot \mathbb{Z}[\chi] /\left\langle 1+\chi+\cdots+\chi^{N-1}\right\rangle \cong 4 \cdot R_{\widehat{\mathbb{Z}}_{N}}$

$$
8 \cdot f_{k}^{\prime} \cdot f \cdot M^{2+2 \operatorname{deg}\left(q_{\bar{t}}\right)} \cdot q_{\bar{t}}\left(f^{2}\right) \in 4 \cdot \mathbb{Z}[\chi] /\left\langle 1+\chi+\cdots+\chi^{N-1}\right\rangle \cong 4 \cdot R_{\widehat{\mathbb{Z}}_{N}} .
$$

Let $z \in \mathbb{Z}$ with $z \cdot M^{3+2 \operatorname{deg}\left(q_{t}\right)+c} \equiv 1 \bmod 2^{K}$. We define $\bar{t}_{4 i}^{\prime}:=z \cdot M^{3+2 \operatorname{deg}\left(q_{\bar{t}}\right)+c}$. $\bar{t}_{4 i} \in \mathbb{Z}(1 \leq i \leq c)$ and conclude

$$
\bar{t}_{4 i}^{\prime} \equiv \bar{t}_{4 i} \equiv \mathbf{t}_{4 i}\left(\left(p_{2^{K}}^{N}\right)^{!}\left(x_{(2)}\right)\right)=\mathbf{t}_{4 i}\left(x_{(2)}\right) \bmod 2^{K}, \quad \bar{t}_{4 i}^{\prime} \equiv 0 \bmod M^{c} .
$$

Proposition 3.15 tells us that $\left[\widetilde{\rho}_{N}\right]\left(x_{(2)}\right) \in \mathbb{Q}_{\widehat{\mathbb{Z}}_{N}}^{(-1)^{d}} / 4 \cdot R_{\widehat{\mathbb{Z}}_{N}}^{(-1)^{d}}$ is given by

$$
\begin{aligned}
d=2 e:\left[\widetilde{\rho}_{N}\right]\left(x_{(2)}\right) & =\sum_{i=1}^{e-1} 8 \cdot \bar{t}_{4 i}^{\prime} \cdot f_{k}^{\prime} \cdot f^{d-2 i-2} \cdot\left(f^{2}-1\right) \\
& =z \cdot M^{c} \cdot 8 \cdot f_{k}^{\prime} \cdot\left(f^{2}-1\right) \cdot M^{3+2 \operatorname{deg}\left(q_{\bar{t}}\right)} \cdot q_{\bar{t}}\left(f^{2}\right) \in 4 \cdot R_{\widehat{\mathbb{Z}}_{N}} \\
d=2 e+1:\left[\widetilde{\rho}_{N}\right]\left(x_{(2)}\right) & =\sum_{i=1}^{e-1} 8 \cdot \bar{t}_{4 i}^{\prime} \cdot f_{k}^{\prime} \cdot f^{d-2 i-2} \cdot\left(f^{2}-1\right)+8 \cdot \bar{t}_{4 e}^{\prime} \cdot f_{k}^{\prime} \cdot f \\
& =z \cdot M^{c+1} \cdot 8 \cdot f_{k}^{\prime} \cdot f \cdot M^{2+2 \operatorname{deg}\left(q_{\bar{t}}\right)} \cdot q_{\bar{t}}\left(f^{2}\right) \in 4 \cdot R_{\widehat{\mathbb{Z}}_{N}} .
\end{aligned}
$$

Hence we have $x_{(2)} \in \operatorname{ker}\left(\left.\left[\widetilde{\rho}_{N}\right]\right|_{\widetilde{\mathcal{N}}\left(L_{N}^{2 d-1}\left(\alpha_{k}\right)\right)_{(2)}}\right)$. 


\section{THE SUSPENSION HOMOMORPHISM}

The suspension homomorphism

$$
\Sigma: \mathcal{S}^{s}\left(L^{2 d-1}\left(\alpha_{k}\right)\right) \rightarrow \mathcal{S}^{s}\left(L^{2 d+1}\left(\alpha_{k}\right)\right)
$$

was already mentioned in section 2, see also Wal99, chapter 14]. It is an interesting question on its own to understand this map. It was also used as an important tool in Wal99, chapter 14] and LdM71 to obtain the calculation of the structure sets of lens spaces when $N=2$ and $N$ odd. We were able to perform our calculations essentially without the use of this map. On the other hand, understanding of $\Sigma$ enables us to give a description of the torsion invariants of fake lens spaces in the next section.

Studying $\Sigma$ is a problem closely related to studying the splitting of simple homotopy equivalences along submanifolds. Recall that a simple homotopy equivalence $h: M^{n} \rightarrow X^{n}$ of manifolds is called split along a locally flat submanifold $Y^{n-q} \subset X^{n}$ if it is transverse to it and the restrictions $h \mid: h^{-1}(Y) \rightarrow Y$ and $h \mid: M \backslash h^{-1}(Y) \rightarrow X \backslash Y$ are simple homotopy equivalences. We also say that $h$ can be made split if it is homotopic to a split map. The question whether a simple homotopy equivalence can be made split is called a splitting problem. It may be obstructed as we discuss below.

Before that notice that if $h: L \rightarrow L^{2 d-1}\left(\alpha_{k}\right)$ is a simple homotopy equivalence representing an element $x \in \mathcal{S}^{s}\left(L^{2 d-1}\left(\alpha_{k}\right)\right)$ then the suspension $\Sigma(x) \in$ $\mathcal{S}^{s}\left(L^{2 d+1}\left(\alpha_{k}\right)\right)$ is split along $L^{2 d-1}\left(\alpha_{k}\right)$. The converse is also true Wal99. Therefore studying $\Sigma$ is equivalent to studying the splitting problems with the target $L^{2 d+1}\left(\alpha_{k}\right)$ along the embedded $L^{2 d-1}\left(\alpha_{k}\right) \subset L^{2 d+1}\left(\alpha_{k}\right)$.

There is a general obstruction theory for the splitting problems, the obstruction groups are the so-called $L S$-groups which are renamed $L N$-groups in the special case when the inclusion $Y \subset X$ induces an isomorphism on the fundamental groups. They depend on the homomorphism $\pi_{1}(X \backslash Y) \rightarrow \pi_{1}(X)$, the dimension $n-q$ and the codimension $q$ (which is usually not indicated in the notation). In fact, in our case $L^{2 d-1}\left(\alpha_{k}\right) \subset L^{2 d+1}\left(\alpha_{k}\right)$ there is an exact sequence (see [Ran81, section 7.2]):

$\cdots \rightarrow L N_{2 d}\left(\mathbb{Z} \rightarrow \mathbb{Z}_{N}\right) \rightarrow \mathcal{S}^{s}\left(L^{2 d-1}\left(\alpha_{k}\right)\right) \stackrel{\Sigma}{\rightarrow} \mathcal{S}^{s}\left(L^{2 d+1}\left(\alpha_{k}\right)\right) \rightarrow L N_{2 d-1}\left(\mathbb{Z} \rightarrow \mathbb{Z}_{N}\right) \rightarrow \cdots$

The $L N$-groups were extensively studied in [LdM71, Wal99], Ran81]. However, we will not directly need these calculations. We will only use the calculations of Wa199 and LdM71 when $N=2$ and $N$ is odd. These calculations are obtained using the $L N$-groups $L N_{*}\left(\mathbb{Z} \rightarrow \mathbb{Z}_{N^{\prime}}\right)$ when $N^{\prime}=2$, so in fact we use them indirectly. The point is that in order to obtain results for general $N \geq 2$ only the groups $L N_{*}\left(\mathbb{Z} \rightarrow \mathbb{Z}_{N^{\prime}}\right)$ are needed where $N^{\prime}=2$. In particular we do not need the complicated calculation of [Ran81, section 7.8].

In Remark 6.9 we also describe a relation of invariants we obtain to the so-called Browder-Livesay invariants.

Theorem 6.1. Let $G=\mathbb{Z}_{N}$ with $N=2^{K} \cdot M, M$ odd and $K \geq 1$ and $e \geq 1$. Then we have for the map

$$
\Sigma: \mathcal{S}^{s}\left(L_{N}^{4 e+1}\left(\alpha_{k}\right)\right) \rightarrow \mathcal{S}^{s}\left(L_{N}^{4 e+3}\left(\alpha_{k}\right)\right)
$$

that:

(1) it is split-injective.

(2) $\mathcal{S}^{s}\left(L_{N}^{4 e+3}\left(\alpha_{k}\right)\right) \cong \operatorname{im}(\Sigma) \oplus \mathbb{Z}(\sigma)$ where $\sigma \in \widetilde{L}_{4 e+4}^{s}(G) \subset \mathcal{S}^{s}\left(L_{N}^{4 e+3}\left(\alpha_{k}\right)\right)$ is defined by $\widetilde{\rho}(\sigma)=8 \in 4 \cdot R_{\widetilde{G}}^{+}$.

(3) $\operatorname{im}(\Sigma)=\operatorname{ker}\left(\mathcal{S}^{s}\left(L_{N}^{4 e+3}\left(\alpha_{k}\right)\right) \stackrel{\widetilde{\rho}}{\rightarrow} \mathbb{Q} R_{\widehat{G}} \stackrel{\chi \mapsto-1}{\longrightarrow} \mathbb{Q}\right)$. 
Theorem 6.2. Let $G=\mathbb{Z}_{N}$ with $N=2^{K} \cdot M, M$ odd and $K \geq 1, e \geq 2$. Then we have for the map

$$
\Sigma: \mathcal{S}^{s}\left(L_{N}^{4 e-1}\left(\alpha_{k}\right)\right) \rightarrow \mathcal{S}^{s}\left(L_{N}^{4 e+1}\left(\alpha_{k}\right)\right)
$$

that:

(1) $\operatorname{ker}(\Sigma)=\mathbb{Z}(\omega)$ where $\omega \in \widetilde{L}_{4 e}^{s}\left(\mathbb{Z}_{N}\right) \subset \mathcal{S}^{s}\left(L_{N}^{4 e-1}\left(\alpha_{k}\right)\right)$ is defined by

$$
\widetilde{\rho}(\omega)=16 \cdot\left(1+\chi^{2}+\cdots+\chi^{N-2}\right) \in 4 \cdot R_{\widehat{G}}^{+} .
$$

(2) $\mathcal{S}^{s}\left(L_{N}^{4 e+1}\left(\alpha_{k}\right)\right) \cong \operatorname{im}(\Sigma) \oplus \mathbb{Z}_{2}\left(\mu_{4 e-2}\right)$ where $\mu_{4 e-2} \in \mathcal{S}^{s}\left(L_{N}^{4 e+1}\left(\alpha_{k}\right)\right)$ is defined by $\widetilde{\rho}\left(\mu_{4 e-2}\right)=0, \mathbf{t}_{(\text {odd })}\left(\eta\left(\mu_{4 e-2}\right)\right)=0, \mathbf{t}_{4 e-2}\left(\eta\left(\mu_{4 e-2}\right)\right)=1$ and $\mathbf{t}_{(2 i)}\left(\eta\left(\mu_{4 e-2}\right)\right)=0$ for $i \neq 2 e-1$.

(3) $\operatorname{im}(\Sigma)=\operatorname{ker}\left(\mathcal{S}^{s}\left(L_{N}^{4 e+1}\left(\alpha_{k}\right)\right) \stackrel{\mathbf{t}_{4 e-2} \circ \eta}{\longrightarrow} \mathbb{Z}_{2}\right)$.

Remark 6.3. The two theorems can be summarized in the following exact sequences (when $e \geq 1$ or $e \geq 2$ respectively):

$$
\begin{aligned}
& 0 \rightarrow \mathcal{S}^{s}\left(L_{N}^{4 e+1}\left(\alpha_{k}\right)\right) \stackrel{\Sigma}{\rightarrow} \mathcal{S}^{s}\left(L_{N}^{4 e+3}\left(\alpha_{k}\right)\right) \rightarrow \mathbb{Z}(\sigma) \rightarrow 0 \\
& 0 \rightarrow \mathbb{Z}(\omega) \rightarrow \mathcal{S}^{s}\left(L_{N}^{4 e-1}\left(\alpha_{k}\right)\right) \stackrel{\Sigma}{\rightarrow} \mathcal{S}^{s}\left(L_{N}^{4 e+1}\left(\alpha_{k}\right)\right) \rightarrow \mathbb{Z}_{2}\left(\mu_{4 e-2}\right) \rightarrow 0 .
\end{aligned}
$$

\subsection{Proof of Theorem 6.1}

Lemma 6.4. The restriction map

$$
\text { res: } \tilde{\mathcal{N}}\left(L_{N}^{4 e+3}\left(\alpha_{k}\right)\right) \rightarrow \tilde{\mathcal{N}}\left(L_{N}^{4 e+1}\left(\alpha_{k}\right)\right)
$$

is an isomorphism.

Proof. The following diagram implies that the restriction map is surjective.

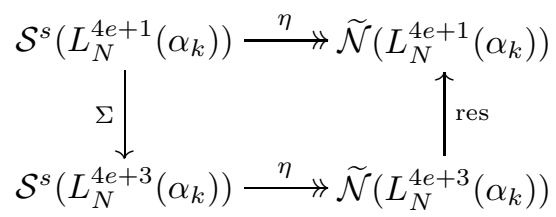

The proof of injectivity is more difficult. We first consider the case $N$ odd. By an Atiyah-Hirzebruch spectral sequence argument Wall analyzed that the order of the group $\widetilde{\mathcal{N}}\left(L_{N}^{2 d-1}\left(\alpha_{k}\right)\right) \cong K O\left(L^{2 d-1}(\alpha)\right) \otimes \mathbb{Z}\left[\frac{1}{2}\right]$ is $N^{c}$ with $c=\lfloor(d-1) / 2\rfloor$. Hence we have $\left|\tilde{\mathcal{N}}\left(L_{N}^{4 e+3}\left(\alpha_{k}\right)\right)\right|=\left|\tilde{\mathcal{N}}\left(L_{N}^{4 e+1}\left(\alpha_{k}\right)\right)\right|$ and conclude that the restriction map is bijective for $N$ odd. For $N$ even we have the following diagram.

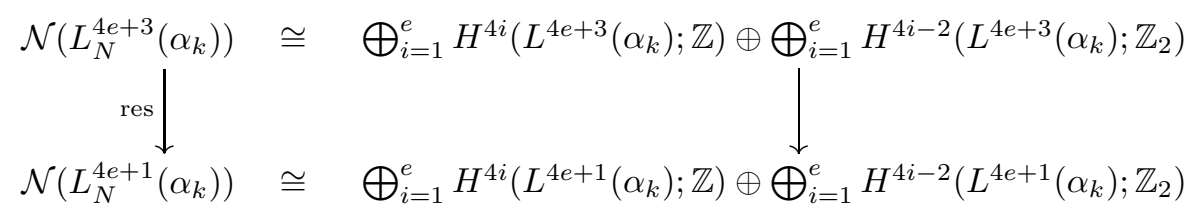

The map on the right hand side is induced by the inclusion $L^{4 e+1}\left(\alpha_{k}\right) \subset L^{4 e+3}\left(\alpha_{k}\right)$ and is hence an isomorphism. This shows that res: $\widetilde{\mathcal{N}}\left(L_{N}^{4 e+3}\left(\alpha_{k}\right)\right) \rightarrow \widetilde{\mathcal{N}}\left(L_{N}^{4 e+1}\left(\alpha_{k}\right)\right)$ is an isomorphism for $N$ even. It remains to consider the case $N=M \cdot 2^{K}$ with $M>1$ odd and $K \geq 1$. The diagram

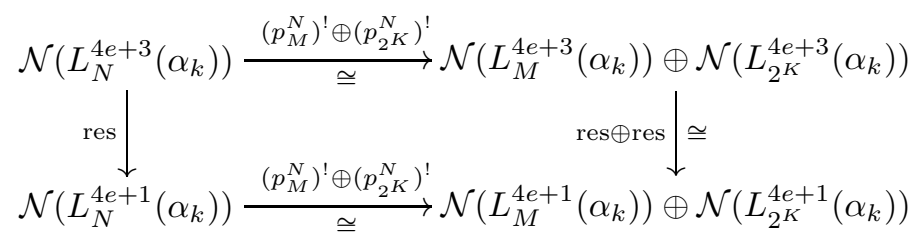

implies that the restriction map is an isomorphism. 
Proof of Theorem 6.1.

(1) Let $x \in \operatorname{ker}(\Sigma)$. Then

$$
\eta(x)=\operatorname{res} \circ \eta \circ \Sigma(x)=0 \quad \text { and hence } \quad x \in \widetilde{L}_{4 e+2}^{s}(G) \subset \mathcal{S}^{s}\left(L_{N}^{4 e+1}\left(\alpha_{k}\right)\right) .
$$

Further

$$
0=\widetilde{\rho}(\Sigma(x))=f \cdot \widetilde{\rho}(x) .
$$

By $[$ MW08, Lemma 5.6] we obtain $\widetilde{\rho}(x)=0$. These two facts together imply $x=0$.

(2) First we show

$$
\mathcal{S}^{s}\left(L_{N}^{4 e+3}\left(\alpha_{k}\right)\right) \cong \operatorname{im}(\Sigma)+\mathbb{Z}(\sigma)
$$

Let $x \in \mathcal{S}^{s}\left(L_{N}^{4 e+3}\left(\alpha_{k}\right)\right)$. Choose $y \in \mathcal{S}^{s}\left(L_{N}^{4 e+1}\left(\alpha_{k}\right)\right)$ such that $\eta(y)=$ res $\circ \eta(x)$. Then we have res $\circ \eta(x-\Sigma(y))=0$ and by Lemma 6.4 also $\eta(x-\Sigma(y))=0$ and hence $x-\Sigma(y) \in \widetilde{L}_{4 e+4}^{s}(G) \subset \mathcal{S}^{s}\left(L_{N}^{4 e+3}\left(\alpha_{k}\right)\right)$.

Let $8 \cdot z$ where $z \in \mathbb{Z}$ be the image of $x-\Sigma(y)$ under the composition

$$
\mathcal{S}^{s}\left(L_{N}^{4 e+3}\left(\alpha_{k}\right)\right) \stackrel{\widetilde{\rho}}{\rightarrow} 4 \cdot R_{\widehat{G}}^{+} \stackrel{\chi \mapsto-1}{\longrightarrow} 8 \cdot \mathbb{Z}
$$

Then we have that

$$
\widetilde{\rho}(x-\Sigma(y)-z \cdot \sigma) \in 4 \cdot R_{\widehat{G}}^{+} \subset 4 \cdot \mathbb{Z}[\chi] /\left\langle 1+\chi+\cdots+\chi^{N-1}\right\rangle
$$

is divisible by $1+\chi$. Now we need the following lemma.

Lemma 6.5. Let $u \in 4 \cdot R_{\widehat{G}}^{+}$be an element which maps to 0 under the map 4 . $R_{\widehat{G}}^{+} \stackrel{\chi \mapsto-1}{\longrightarrow} 8 \cdot \mathbb{Z}$. Then there exists $a \in 4 \cdot R_{\widehat{G}}^{-}$such that

$$
u=f \cdot a \text {. }
$$

Proof. It is enough to prove the lemma for

$$
u=4 \cdot\left(\chi^{k}+\chi^{-k}\right)+8 \cdot(-1)^{k+1},
$$

where $k=1, \ldots, N / 2$. We have $u=(1+\chi) \cdot v$ where

$$
\begin{aligned}
v & =4 \cdot\left(\chi^{k-1}-\chi^{k-2}+\cdots+(-1)^{k+1}\right)+ \\
& +4 \cdot\left(\chi^{-k}-\chi^{-k+1}+\cdots+(-1)^{k+1} \cdot \chi^{-1}\right) .
\end{aligned}
$$

Setting $a=(1-\chi) \cdot v$ one easily verifies the desired equation as well as the fact that $a \in 4 \cdot R_{\widehat{G}}^{-}$.

Applying the above lemma to $u=\widetilde{\rho}(x-\Sigma(y)-z \cdot \sigma)$ we find an element $y^{\prime} \in$ $\widetilde{L}_{4 e+2}^{s}(G) \subset \mathcal{S}^{s}\left(L_{N}^{4 e+1}\left(\alpha_{k}\right)\right)$ such that $\widetilde{\rho}\left(y^{\prime}\right)=a$.

Using Lemma 6.4 we see that $\eta \circ \Sigma\left(y^{\prime}\right)=0$. Further $\widetilde{\rho}\left(x-\Sigma\left(y+y^{\prime}\right)-z \cdot \sigma\right)=0$. Hence

$$
x=\Sigma\left(y+y^{\prime}\right)+z \cdot \sigma \in \operatorname{im}(\Sigma)+\mathbb{Z}(\sigma) .
$$

To see that we obtain the direct sum it is enough to observe that under the homomorphism

$$
\mathcal{S}^{s}\left(L_{N}^{4 e+3}\left(\alpha_{k}\right)\right) \stackrel{\widetilde{\rho}}{\rightarrow} \mathbb{Q} R_{\widehat{G}} \stackrel{\chi \mapsto-1}{\longrightarrow} \mathbb{Q}
$$

the subgroup $\operatorname{im}(\Sigma)$ is mapped to 0 (because $\widetilde{\rho}(\Sigma(y))=f \cdot \widetilde{\rho}(y)$ ) and that $\sigma$ is mapped to 8 by definition.

(3) This follows from the proof of the previous point. 


\subsection{Proof of Theorem 6.2 .}

Lemma 6.6. Let $G=\mathbb{Z}_{N}$ with $N=2^{K} \cdot M, M$ odd and $K \geq 1$. Then

$$
\mathbf{t}_{4 e-2} \circ \eta \circ \Sigma(x)=0 \text { for all } x \in \mathcal{S}^{s}\left(L_{N}^{4 e-1}\left(\alpha_{k}\right)\right) \text {. }
$$

Proof. For $x \in \mathcal{S}^{s}\left(L_{N}^{4 e-1}\left(\alpha_{k}\right)\right)$ we have

$$
\mathbf{t}_{4 e-2} \circ \eta \circ \Sigma(x)=\mathbf{t}_{4 e-2} \circ\left(p_{2}^{N}\right)^{!} \circ \eta \circ \Sigma(x)=\mathbf{t}_{4 e-2} \circ \eta \circ \Sigma\left(\left(p_{2}^{N}\right)^{!}(x)\right) .
$$

Therefore, it suffices to prove the statement for $N=2$. López de Medrano shows in [LdM71, chapter IV] that $\mathcal{S}^{s}\left(L_{2}^{4 e+1}\left(\alpha_{k}\right)\right) / \operatorname{im}(\Sigma) \cong \mathbb{Z}_{2}$ where the isomorphism is induced by the map $\mathbf{t}_{4 e-2} \circ \eta$. In particular, we have $\mathbf{t}_{4 e-2} \circ \eta \circ \Sigma(x)=0$ for all $x \in \mathcal{S}^{s}\left(L_{2}^{4 e-1}\left(\alpha_{k}\right)\right)$.

Lemma 6.7. Let $G=\mathbb{Z}_{N}$ with $N=2^{K} \cdot M, M$ odd and $K \geq 1$. Let $\tau_{N} \in$ $\widetilde{L}_{4 e}^{s}\left(\mathbb{Z}_{N}\right) \subset \mathcal{S}^{s}\left(L_{N}^{4 e-1}\left(\alpha_{k}\right)\right)$ be defined by

$$
\operatorname{G-sign}\left(\tau_{N}\right)=2^{\max \{4-K, 2\}} \cdot\left(1+\chi^{2}+\cdots+\chi^{N-2}\right) \in 4 \cdot R_{\widehat{G}}^{+} .
$$

Then $\Sigma\left(\tau_{N}\right)$ has the following properties:

(1) $\widetilde{\rho}\left(\Sigma\left(\tau_{N}\right)\right)=0$.

(2) $\mathbf{t}_{2 i} \circ \eta\left(\Sigma\left(\tau_{N}\right)\right)=0$ if $i \neq 2 e$.

(3) $\mathbf{t}_{4 e} \circ \eta\left(\Sigma\left(\tau_{N}\right)\right)\left\{\begin{array}{ll}=1 \in \mathbb{Z}_{2} & K=1 \\ \in\left\{2^{K-2}, 3 \cdot 2^{K-2}\right\} \subset \mathbb{Z}_{2^{K}} & K \geq 2\end{array}\right.$.

(4) $\mathbf{t}_{(\text {odd })} \circ \eta\left(\Sigma\left(\tau_{N}\right)\right)=0$.

Proof. (1) $\widetilde{\rho}\left(\Sigma\left(\tau_{N}\right)\right)=f \cdot \mathrm{G}-\operatorname{sign}\left(\tau_{N}\right)=0$ because

$$
(1+\chi) \cdot\left(1+\chi^{2}+\cdots+\chi^{N-2}\right)=0 \in \mathbb{Q} R_{\widehat{G}} .
$$

(2) res $\circ \eta\left(\Sigma\left(\tau_{N}\right)\right)=\eta\left(\tau_{N}\right)=0$ implies $\mathbf{t}_{2 i} \circ \eta\left(\Sigma\left(\tau_{N}\right)\right)=0$ if $i \notin\{2 e-1,2 e\}$. Lemma 6.6 implies $\mathbf{t}_{4 e-2} \circ \eta\left(\Sigma\left(\tau_{N}\right)\right)=0$.

(3) First we consider the case $N=2$. In LdM71, chapter IV] López de Medrano shows $\mathcal{S}^{s}\left(L_{2}^{4 e+1}\left(\alpha_{k}\right)\right) / \operatorname{im}(\Sigma) \cong \mathbb{Z}_{2}$ where the isomorphism is induced by the map $\mathbf{t}_{4 e-2} \circ \eta$. Let $x \in \mathcal{S}^{s}\left(L_{2}^{4 e+1}\left(\alpha_{k}\right)\right)$ be given by $\widetilde{\rho}(x)=0, \mathbf{t}_{2 i}(\eta(x))=0$ for $i \neq 2 e$ and $\mathbf{t}_{4 e}(\eta(x))=1 \in \mathbb{Z}_{2}$. Because of the result of López de Medrano there exists an element $y \in \mathcal{S}^{s}\left(L_{2}^{4 e-1}\left(\alpha_{k}\right)\right)$ with $\Sigma(y)=x$. Since

$$
\eta(y)=\operatorname{res}(\eta(\Sigma(y)))=\operatorname{res}(\eta(x))=0
$$

holds, we obtain $y \in \widetilde{L}_{4 e}^{s}\left(\mathbb{Z}_{2}\right)=\mathbb{Z}\left(\tau_{2}\right)$. Therefore, we can write $y=m \cdot \tau_{2}$ with $m \in \mathbb{Z}$. We conclude

$$
m \cdot \mathbf{t}_{4 e} \circ \eta\left(\Sigma\left(\tau_{2}\right)\right)=\mathbf{t}_{4 e} \circ \eta(\Sigma(y))=\mathbf{t}_{4 e} \circ \eta(x)=1 \in \mathbb{Z}_{2}
$$

and hence $\mathbf{t}_{4 e} \circ \eta\left(\Sigma\left(\tau_{2}\right)\right)=1 \in \mathbb{Z}_{2}$.

Next we prove the statement for $N=2^{K}$ by induction. We have already checked the case $K=1$. We proceed with the induction step $K \rightarrow K+1$. We have to show $\mathbf{t}_{4 e} \circ \eta\left(\Sigma\left(\tau_{2^{K+1}}\right)\right) \in\left\{2^{K-1}, 3 \cdot 2^{K-1}\right\} \subset \mathbb{Z}_{2^{K+1}}$ which is equivalent to

$$
\operatorname{pr} \circ \mathbf{t}_{4 e} \circ \eta\left(\Sigma\left(\tau_{2}{ }^{K+1}\right)\right)=2^{K-1} \in \mathbb{Z}_{2^{K}}
$$

with pr: $\mathbb{Z}_{2^{K+1}} \rightarrow \mathbb{Z}_{2^{K}}, 1 \mapsto 1$. Notice that

$$
\left(p_{2^{K}}^{2^{K+1}}\right)^{!}\left(\tau_{2^{K+1}}\right)= \begin{cases}\tau_{2} & K=1 \\ 2 \cdot \tau_{2^{K}} & K \geq 2\end{cases}
$$

because

$$
\eta\left(\left(p_{2^{K}}^{2^{K+1}}\right)^{!}\left(\tau_{2^{K+1}}\right)\right)=\left(p_{2^{K}}^{2^{K+1}}\right)^{!}\left(\eta\left(\tau_{2^{K+1}}\right)\right)=0= \begin{cases}\eta\left(\tau_{2}\right) & K=1 \\ \eta\left(2 \cdot \tau_{2^{K}}\right) & K \geq 2\end{cases}
$$


and

$$
\begin{aligned}
\widetilde{\rho}\left(\left(p_{2^{K}}^{2^{K+1}}\right)^{!}\left(\tau_{2^{K+1}}\right)\right) & =2^{\max \{3-K, 2\}} \cdot\left(1+\chi^{2}+\chi^{4}+\cdots+\chi^{2^{K+1}-2}\right) \\
& =2^{\max \{3-K, 2\}+1} \cdot\left(1+\chi^{2}+\chi^{4}+\cdots+\chi^{2^{K}-2}\right) \\
& = \begin{cases}\widetilde{\rho}\left(\tau_{2}\right) & K=1 \\
\widetilde{\rho}\left(2 \cdot \tau_{2^{K}}\right) & K \geq 2\end{cases}
\end{aligned}
$$

hold. Using this result and the induction assumption we conclude

$$
\begin{aligned}
\operatorname{pr} \circ \mathbf{t}_{4 e} \circ \eta\left(\Sigma\left(\tau_{2^{K+1}}\right)\right) & =\mathbf{t}_{4 e} \circ \eta\left(\Sigma\left(\left(p_{2^{K}}^{2^{K+1}}\right) !\left(\tau_{2^{K+1}}\right)\right)\right) \\
& = \begin{cases}\mathbf{t}_{4 e} \circ \eta\left(\Sigma\left(\tau_{2}\right)\right) & K=1 \\
\mathbf{t}_{4 e} \circ \eta\left(\Sigma\left(2 \cdot \tau_{2}\right)\right) & K \geq 2\end{cases} \\
& =2^{K-1} \in \mathbb{Z}_{2^{K}} .
\end{aligned}
$$

It remains to show the statement for $N=2^{K} \cdot M$ with $M>1$. We have the equation $\left(p_{2^{K}}^{N}\right) !\left(\tau_{N}\right)=M \cdot \tau_{2^{K}}$ because

$$
\eta\left(\left(p_{2^{K}}^{N}\right)^{!}\left(\tau_{N}\right)\right)=\left(p_{2^{K}}^{N}\right)^{!}\left(\eta\left(\tau_{N}\right)\right)=0=\eta\left(M \cdot \tau_{2^{K}}\right)
$$

and

$$
\begin{aligned}
\widetilde{\rho}\left(\left(p_{2^{K}}^{N}\right)^{!}\left(\tau_{N}\right)\right) & =2^{\max \{4-K, 2\}} \cdot\left(1+\chi^{2}+\chi^{4}+\cdots+\chi^{N-2}\right) \\
& =2^{\max \{4-K, 2\}} \cdot M \cdot\left(1+\chi^{2}+\chi^{4}+\cdots+\chi^{2^{K}-2}\right) \\
& =\widetilde{\rho}\left(M \cdot \tau_{2^{K}}\right)
\end{aligned}
$$

hold. We conclude

$$
\begin{aligned}
\mathbf{t}_{4 e} \circ \eta\left(\Sigma\left(\tau_{N}\right)\right) & =\mathbf{t}_{4 e} \circ\left(p_{2^{K}}^{N}\right)^{!} \circ \eta\left(\Sigma\left(\tau_{N}\right)\right) \\
& =\mathbf{t}_{4 e} \circ \eta\left(\Sigma\left(\left(p_{2^{K}}^{N}\right)^{!}\left(\tau_{N}\right)\right)\right) \\
& =M \cdot \mathbf{t}_{4 e} \circ \eta\left(\Sigma\left(\tau_{2^{K}}\right)\right) \\
& \begin{cases}=1 \in \mathbb{Z}_{2} & K=1 \\
\in\left\{2^{K-2}, 3 \cdot 2^{K-2}\right\} \subset \mathbb{Z}_{2^{K}} & K \geq 2\end{cases}
\end{aligned}
$$

(4) We have $\left(p_{M}^{N}\right) !\left(\tau_{N}\right)=0$ because

$$
\begin{aligned}
\widetilde{\rho}\left(\left(p_{M}^{N}\right)^{!}\left(\tau_{N}\right)\right) & =2^{\max \{4-K, 2\}} \cdot\left(1+\chi^{2}+\chi^{4}+\cdots+\chi^{N-2}\right) \\
& =2^{\max \{4-K, 2\}+K-1} \cdot\left(1+\chi+\chi^{2}+\cdots+\chi^{M-1}\right) \\
& =0
\end{aligned}
$$

and $\eta\left(\left(p_{M}^{N}\right)^{!}\left(\tau_{N}\right)\right)=\left(p_{M}^{N}\right) !\left(\eta\left(\tau_{N}\right)\right)=0$. We conclude

$$
\left(p_{M}^{N}\right) !\left(\eta\left(\Sigma\left(\tau_{N}\right)\right)\right)=\eta\left(\Sigma\left(\left(p_{M}^{N}\right) !\left(\tau_{N}\right)\right)\right)=0
$$

and hence $\mathbf{t}_{(\text {odd })} \circ \eta\left(\Sigma\left(\tau_{N}\right)\right)=0$.

Lemma 6.8. Let $G=\mathbb{Z}_{N}$. If $t \in \mathbb{Z}$ satisfies $8 \cdot t \cdot f_{k} \in 4 \cdot R_{\widehat{G}}$ then $N$ divides $4 \cdot t$.

Proof. Notice that any element in $R_{\widehat{G}}$ respectively in $\mathbb{Q} R_{\widehat{G}}$ can be uniquely written as $\sum_{l=0}^{N-2} c_{l} \cdot \chi^{l \cdot k}$ with $c_{l} \in \mathbb{Z}$ respectively in. $\mathbb{Q}$. We have

$$
f_{k}=1-\frac{2}{N}+\sum_{l=1}^{N-2}\left(2-\frac{2+2 \cdot l}{N}\right) \cdot \chi^{l \cdot k}
$$

Hence $8 \cdot t \cdot f_{k} \in 4 \cdot R_{\widehat{G}}$ leads to an equation

$$
8 \cdot t \cdot\left(1-\frac{2}{N}\right)+\sum_{l=1}^{N-2} 8 \cdot t \cdot\left(2-\frac{2+2 \cdot l}{N}\right) \cdot \chi^{l \cdot k}=\sum_{l=0}^{N-2} 4 \cdot c_{l} \cdot \chi^{l \cdot k}
$$


with $c_{l} \in \mathbb{Z}$. A comparison of the coefficients of $\chi^{0}$ gives $8 \cdot t \cdot(1-2 / N)=4 \cdot c_{0}$ and hence $2 \cdot t \cdot(1-2 / N) \in \mathbb{Z}$. This implies $4 \cdot t / N \in \mathbb{Z}$.

Proof of Theorem 6.2. (1) By Lemma 6.7 $\omega=2^{\min \{K, 2\}} \cdot \tau_{N}$ has the properties $\widetilde{\rho}(\Sigma(\omega))=0$ and $\eta(\Sigma(\omega))=0$. This implies $\Sigma(\omega)=0$ and hence $\mathbb{Z}(\omega) \subseteq \operatorname{ker}(\Sigma)$. We want to prove equality. Let $x \in \operatorname{ker}(\Sigma)$. We conclude $x \in \widetilde{L}_{4 e}^{s}\left(\mathbb{Z}_{N}\right) \subset \mathcal{S}^{s}\left(L_{N}^{4 e-1}\left(\alpha_{k}\right)\right)$ because $\eta(x)=$ res $\circ \eta \circ \Sigma(x)=0$. We have $f \cdot \mathrm{G}-\operatorname{sign}(x)=\widetilde{\rho}(\Sigma(x))=0$ and hence $\mathrm{G}-\operatorname{sign}(x) \equiv 0 \bmod 1+\chi^{2}+\cdots+\chi^{N-2}$. This implies that $x$ is a multiple of $\tau_{N}$. Since $\Sigma(x)=0$, we conclude using Lemma 6.7 that $x$ is a multiple of $\omega=2^{\min \{K, 2\}} \cdot \tau_{N}$.

(2) Let $x_{0} \in \mathcal{S}^{s}\left(L_{N}^{4 e+1}\left(\alpha_{k}\right)\right)$. We want to show $x_{0} \in \operatorname{im}(\Sigma)+\mathbb{Z}_{2}\left(\mu_{4 e-2}\right)$. We choose $y_{1} \in \mathcal{S}^{s}\left(L_{N}^{4 e-1}\left(\alpha_{k}\right)\right)$ with $\eta\left(y_{1}\right)=\operatorname{res}\left(\eta\left(x_{0}\right)\right)$. Then $x_{1}:=x_{0}-\Sigma\left(y_{1}\right)$ satisfies

$$
\operatorname{res}\left(\eta\left(x_{1}\right)\right)=\operatorname{res}\left(\eta\left(x_{0}\right)\right)-\operatorname{res}\left(\eta\left(\Sigma\left(y_{1}\right)\right)\right)=\eta\left(y_{1}\right)-\eta\left(y_{1}\right)=0 .
$$

If $M=1$ then we set $y_{2}:=0 \in \widetilde{L}_{4 e}^{s}\left(\mathbb{Z}_{N}\right) \subset \mathcal{S}^{s}\left(L_{N}^{4 e-1}\left(\alpha_{k}\right)\right)$. Otherwise we proceed as follows to define $y_{2}$ : Consider $\Sigma^{-1}\left(\left(p_{M}^{N}\right) !\left(x_{1}\right)\right) \in \mathcal{S}^{s}\left(L_{M}^{4 e-1}\left(\alpha_{k}\right)\right)$. We have $\Sigma^{-1}\left(\left(p_{M}^{N}\right)^{!}\left(x_{1}\right)\right) \in \widetilde{L}_{4 e}^{s}\left(\mathbb{Z}_{M}\right) \subset \mathcal{S}^{s}\left(L_{M}^{4 e-1}\left(\alpha_{k}\right)\right)$ because

$$
\eta\left(\Sigma^{-1}\left(\left(p_{M}^{N}\right)^{!}\left(x_{1}\right)\right)\right)=\operatorname{res}\left(\eta\left(\left(p_{M}^{N}\right)^{!}\left(x_{1}\right)\right)\right)=\left(p_{M}^{N}\right)^{!}\left(\operatorname{res}\left(\eta\left(x_{1}\right)\right)\right)=0 .
$$

We choose $y_{2} \in \widetilde{L}_{4 e}^{s}\left(\mathbb{Z}_{N}\right) \subset \mathcal{S}^{s}\left(L_{N}^{4 e-1}\left(\alpha_{k}\right)\right)$ with $\left(p_{M}^{N}\right)^{!}\left(y_{2}\right)=\Sigma^{-1}\left(\left(p_{M}^{N}\right)^{!}\left(x_{1}\right)\right)$. We set $x_{2}:=x_{1}-\Sigma\left(y_{2}\right)=x_{0}-\Sigma\left(y_{1}+y_{2}\right)$. Notice that

$$
\operatorname{res}\left(\eta\left(x_{2}\right)\right)=\operatorname{res}\left(\eta\left(x_{1}\right)\right)-\operatorname{res}\left(\eta\left(\Sigma\left(y_{2}\right)\right)\right)=0-\eta\left(y_{2}\right)=0
$$

and hence $\mathbf{t}_{2 i}\left(\eta\left(x_{2}\right)\right)=0$ if $i \notin\{2 e-1,2 e\}$. We have $\mathbf{t}_{(o d d)}\left(\eta\left(x_{2}\right)\right)=0$ because $\left(p_{M}^{N}\right)^{!}\left(\eta\left(x_{2}\right)\right)=\left(p_{M}^{N}\right)^{!}\left(\eta\left(x_{1}-\Sigma\left(y_{2}\right)\right)\right)=\eta\left(\left(p_{M}^{N}\right)^{!}\left(x_{1}\right)-\Sigma\left(\left(p_{M}^{N}\right)^{!}\left(y_{2}\right)\right)\right)=\eta(0)=0$. Therefore, $\widetilde{\rho}\left(x_{2}\right)$ is of the shape $\widetilde{\rho}\left(x_{2}\right)=8 \cdot t \cdot f_{k}+r$ with $t \in \mathbb{Z}$ and $r \in 4 \cdot R_{\widehat{G}}^{-}$(see Proposition 3.14). Since $8 \cdot t \cdot f_{k}^{\prime} \in 4 \cdot R_{\widehat{G}}^{+}$and

$$
\begin{aligned}
4 \cdot\left(\chi^{l}-\chi^{N-l}\right)=f \cdot & \left(4 \cdot\left(\chi^{l}+\chi^{N-l}\right)-8 \cdot\left(\chi^{l+1}+\chi^{N-l-1}\right)+\cdots+\right. \\
& \left.(-1)^{N / 2-l-1} \cdot 8 \cdot\left(\chi^{N / 2-1}+\chi^{N / 2+l}\right)+(-1)^{N / 2-l} \cdot 8 \cdot \chi^{N / 2}\right)
\end{aligned}
$$

for $1 \leq l \leq N / 2-1$, there exists $z \in 4 \cdot R_{\widehat{G}}^{+}$with $f \cdot z=\widetilde{\rho}\left(x_{2}\right)$. We define $y_{3}:=\mathrm{G}_{-} \operatorname{sign}^{-1}(z) \in \widetilde{L}_{4 e}^{s}\left(\mathbb{Z}_{N}\right) \subset \mathcal{S}^{s}\left(L_{N}^{4 e-1}\left(\alpha_{k}\right)\right)$ and

$$
x_{3}:=x_{2}-\Sigma\left(y_{3}\right)=x_{0}-\Sigma\left(y_{1}+y_{2}+y_{3}\right) \text {. }
$$

We have

$$
\widetilde{\rho}\left(x_{3}\right)=\widetilde{\rho}\left(x_{2}\right)-\widetilde{\rho}\left(\Sigma\left(y_{3}\right)\right)=\widetilde{\rho}\left(x_{2}\right)-f \cdot z=0 .
$$

Since $\widetilde{\rho}\left(\left(p_{M}^{N}\right)^{!}\left(x_{3}\right)\right)=0$ holds and $\widetilde{\rho}: \mathcal{S}^{s}\left(L_{M}^{4 e+1}\left(\alpha_{k}\right)\right) \rightarrow \mathbb{Q} R_{\widehat{\mathbb{Z}}_{M}}^{-}$is injective, we obtain $\left(p_{M}^{N}\right)^{!}\left(x_{3}\right)=0$. This implies $\left(p_{M}^{N}\right)^{!}\left(\eta\left(x_{3}\right)\right)=0$ and hence $\mathbf{t}_{(\text {odd })}\left(\eta\left(x_{3}\right)\right)=0$. Moreover, we have $\mathbf{t}_{2 i}\left(\eta\left(x_{3}\right)\right)=0$ if $i \notin\{2 e-1,2 e\}$ because

$$
\operatorname{res}\left(\eta\left(x_{3}\right)\right)=\operatorname{res}\left(\eta\left(x_{2}\right)\right)-\operatorname{res}\left(\eta\left(\Sigma\left(y_{3}\right)\right)\right)=0-\eta\left(y_{3}\right)=0 .
$$

Let $t_{4 e-2} \in\{0,1\}$ with $\mathbf{t}_{4 e-2}\left(\eta\left(x_{3}\right)\right)=t_{4 e-2}$. We set

$$
x_{4}:=x_{3}-t_{4 e-2} \cdot \mu_{4 e-2}=x_{0}-\Sigma\left(y_{1}+y_{2}+y_{3}\right)-t_{4 e-2} \cdot \mu_{4 e-2}
$$

and conclude $\widetilde{\rho}\left(x_{4}\right)=0, \mathbf{t}_{(o d d)}\left(\eta\left(x_{4}\right)\right)=0$ and $\mathbf{t}_{2 i}\left(\eta\left(x_{4}\right)\right)=0$ for $i \neq 2 e$. By Proposition 3.14 we have $0=\widetilde{\rho}\left(x_{4}\right)=8 \cdot t \cdot f_{k}+s$ where $s \in 4 \cdot R_{\widehat{G}}^{-}$and $t \in \mathbb{Z}$ with $t \equiv \mathbf{t}_{4 e}\left(\eta\left(x_{4}\right)\right) \bmod 2^{K}, t \equiv 0 \bmod M$. We know by Lemma 6.8 that $2^{\max \{K-2,0\}}$ divides $t$. Hence $\mathbf{t}_{4 e}\left(\eta\left(x_{4}\right)\right)$ is a multiple of $2^{\max \{K-2,0\}}$. By Lemma 6.7 there exists $n_{\tau} \in \mathbb{Z}$ with $\mathbf{t}_{4 e}\left(\eta\left(x_{4}\right)\right)=n_{\tau} \cdot \mathbf{t}_{4 e}\left(\eta\left(\Sigma\left(\tau_{N}\right)\right)\right)$. Using again Lemma 6.7 we conclude that the element

$$
x_{5}:=x_{4}-n_{\tau} \cdot \Sigma\left(\tau_{N}\right)=x_{0}-\Sigma\left(y_{1}+y_{2}+y_{3}+n_{\tau} \cdot \tau_{N}\right)-t_{4 e-2} \cdot \mu_{4 e-2}
$$


satisfies $\widetilde{\rho}\left(x_{5}\right)=0, \mathbf{t}_{(o d d)}\left(\eta\left(x_{5}\right)\right)=0$ and $\mathbf{t}_{2 i}\left(\eta\left(x_{5}\right)\right)=0$ for all $i$. Hence $x_{5}=0$ and $x_{0}=\Sigma\left(y_{1}+y_{2}+y_{3}+n_{\tau} \cdot \tau_{N}\right)+t_{4 e-2} \cdot \mu_{4 e-2} \in \operatorname{im}(\Sigma)+\mathbb{Z}_{2}\left(\mu_{4 e-2}\right)$. This shows $\mathcal{S}^{s}\left(L_{N}^{4 e+1}\left(\alpha_{k}\right)\right)=\operatorname{im}(\Sigma)+\mathbb{Z}_{2}\left(\mu_{4 e-2}\right)$. Notice that $\operatorname{im}(\Sigma) \cap \mathbb{Z}_{2}\left(\mu_{4 e-2}\right)=0$ because of Lemma 6.6 and $\mathbf{t}_{4 e-2}(\eta(a))=1$. Therefore, we have $\mathcal{S}^{s}\left(L_{N}^{4 e+1}\left(\alpha_{k}\right)\right)=$ $\operatorname{im}(\Sigma) \oplus \mathbb{Z}_{2}\left(\mu_{4 e-2}\right)$.

(3) This follows from Lemma 6.6 and assertion (2).

Remark 6.9. The question when is a given element in the image of the suspension map is called a desuspension problem. It was solved for $N=2$ by López de Medrano in LdM71] using the so-called Browder-Livesay invariants, denoted $\sigma$. Depending on the dimension one has $\sigma: \mathcal{S}\left(L_{2}^{4 e-1}(\alpha)\right) \rightarrow \mathbb{Z}$, or $\sigma: \mathcal{S}\left(L_{2}^{4 e+1}(\alpha)\right) \rightarrow \mathbb{Z}_{2}$. In the dimension $4 e+1$ we have $\sigma=\mathbf{t}_{4 e-2} \circ \eta$. López de Medrano also showed in LdM71, section IV.4] that in the dimensions $4 e-1$ the invariant $\sigma$ coincides with the $\widetilde{\rho}$ invariant which is just an integer in the case $N=2$.

Notice that the desuspension obstruction we obtain in the dimensions $4 e-1$ is given by sending $\chi \mapsto-1$ which corresponds to passing to fake projective spaces via the transfer $\left(p_{2}^{N}\right)^{!}$induced by the inclusion $\mathbb{Z}_{2} \subset \mathbb{Z}_{N}$. Therefore our obstructions to desuspension can be obtained by first applying transfer and then taking the Browder-Livesay invariant.

\section{INVARIANTS OF FAKE LENS SPACES}

Our main Theorem 1.2 gives an abstract calculation of the structure sets of fake lens spaces. However, in order to obtain a satisfactory classification one needs in addition to such a calculation a good description of the invariants. In our case we have a good description of the homomorphism into the free abelian factor by the $\widetilde{\rho}$-invariant. But as already indicated in the introduction the main theorem does not yield such a good description for the torsion part.

We make a step towards a remedy of this deficiency. We still need to make a choice of the splitting of the structure set into the free abelian group and the torsion group as stated in Proposition 4.1, see the proof of Theorem 5.1 in [MW08. However, once this choice is made, we obtain, in a special case, an isomorphism of the torsion part where the factors have certain geometric interpretation. It will be an obstruction-theoretic description in a sense that we can interpret certain factor if all the previous factors vanish.

The special case we treat is when $\alpha=\alpha_{k}$. Note that the lens spaces $L_{N}^{2 d-1}\left(\alpha_{k}\right)$ are obtained as joins $L_{N}^{1}\left(\alpha_{k}\right) * L_{N}^{1}\left(\alpha_{1}\right) * \cdots * L_{N}^{1}\left(\alpha_{1}\right)$ and hence they contain sublens spaces $L_{N}^{2 i-1}\left(\alpha_{k}\right)$ for all $i \leq d$. This will be used in the description of the invariants. Roughly speaking one examines the restrictions of the simple homotopy equivalences which represent elements of $\mathcal{S}^{s}\left(L_{N}^{2 d-1}\left(\alpha_{k}\right)\right)_{\text {tors }}$ to the inverse images of these sub-lens spaces.

Proposition 7.1. Let $G=\mathbb{Z}_{N}$ with $N=2^{K} \cdot M, M$ odd and $K \geq 1$, e $\geq 2$. Then we have for the map

$$
\Sigma: \mathcal{S}^{s}\left(L_{N}^{4 e-1}\left(\alpha_{k}\right)\right) \rightarrow \mathcal{S}^{s}\left(L_{N}^{4 e+1}\left(\alpha_{k}\right)\right)
$$

that:

(1) $2^{4-\min \{K, 2 e\}} \cdot\left(1+\chi^{2}+\cdots+\chi^{N-2}\right) \in \operatorname{im}\left(\widetilde{\rho}: \mathcal{S}^{s}\left(L_{N}^{4 e-1}\left(\alpha_{k}\right)\right) \rightarrow \mathbb{Q} R_{\widehat{G}}\right)$.

(2) Let $\nu_{e} \in \mathcal{S}^{s}\left(L_{N}^{4 e-1}\left(\alpha_{k}\right)\right)$ be such that

$$
\widetilde{\rho}\left(\nu_{e}\right)=2^{4-\min \{K, 2 e\}} \cdot\left(1+\chi^{2}+\cdots+\chi^{N-2}\right) .
$$

Then we have

$$
\mathcal{S}^{s}\left(L_{N}^{4 e+1}\left(\alpha_{k}\right)\right)_{\text {tors }} \cong \operatorname{im}\left(\left.\Sigma\right|_{\text {tors }}\right) \oplus \mathbb{Z}_{2^{\min \{K, 2 e\}}}\left(\Sigma\left(\nu_{e}\right)\right) \oplus \mathbb{Z}_{2}\left(\mu_{4 e-2}\right)
$$


where $\mu_{4 e-2} \in \mathcal{S}^{s}\left(L_{N}^{4 e+1}\left(\alpha_{k}\right)\right)$ is defined by $\widetilde{\rho}\left(\mu_{4 e-2}\right)=0, \mathbf{t}_{(\text {odd })}\left(\eta\left(\mu_{4 e-2}\right)\right)=$ $0, \mathbf{t}_{4 e-2}\left(\eta\left(\mu_{4 e-2}\right)\right)=1$ and $\mathbf{t}_{2 i}\left(\eta\left(\mu_{4 e-2}\right)\right)=0$ for $i \neq 2 e-1$.

Proof. Let $l \in \mathbb{Z}$ be the smallest integer such that $2^{l} \cdot\left(1+\chi^{2}+\cdots+\chi^{N-2}\right)$ is in the image $\operatorname{im}\left(\widetilde{\rho}: \mathcal{S}^{s}\left(L_{N}^{4 e-1}\left(\alpha_{k}\right)\right) \rightarrow \mathbb{Q} R_{\widehat{G}}\right)$. Using the transfer $\left(p_{2}^{N}\right)$ ! which sends $\chi \mapsto-1$ we conclude $2^{l} \cdot 2^{K-1} \cdot M \in 8 \cdot \mathbb{Z}$ and hence $l \geq 4-K$. At the end of the proof we show that $l=4-\min \{K, 2 e\}$. Before that we prove the other statements.

Let $\nu_{e} \in \mathcal{S}^{s}\left(L_{N}^{4 e-1}\left(\alpha_{k}\right)\right)$ be such that $\widetilde{\rho}\left(\nu_{e}\right)=2^{l} \cdot\left(1+\chi^{2}+\cdots+\chi^{N-2}\right)$. Since $\widetilde{\rho}\left(\Sigma\left(\nu_{e}\right)\right)=f \cdot \widetilde{\rho}\left(\nu_{e}\right)=0$ we obtain $\Sigma\left(\nu_{e}\right) \in \mathcal{S}^{s}\left(L_{N}^{4 e+1}\left(\alpha_{k}\right)\right)_{\text {tors }}$.

We first show that

$$
\mathcal{S}^{s}\left(L_{N}^{4 e+1}\left(\alpha_{k}\right)\right)_{\text {tors }}=\operatorname{im}\left(\left.\Sigma\right|_{\text {tors }}\right)+\mathbb{Z}_{\left.2^{\min \{K, 2 e}\right\}}\left(\Sigma\left(\nu_{e}\right)\right)+\mathbb{Z}_{2}\left(\mu_{4 e-2}\right) .
$$

Abbreviate $\nu=\nu_{e}$ and $\mu=\mu_{4 e-2}$ and let $y \in \mathcal{S}^{s}\left(L_{N}^{4 e+1}\left(\alpha_{k}\right)\right)_{\text {tors }}$. Since we know $\mathcal{S}^{s}\left(L_{N}^{4 e+1}\left(\alpha_{k}\right)\right)=\operatorname{im}(\Sigma) \oplus \mathbb{Z}_{2}(\mu)$, we have $y=\Sigma(x)+n_{\mu} \cdot \mu$ for some $x \in \mathcal{S}^{s}\left(L_{N}^{4 e+1}\left(\alpha_{k}\right)\right)$.

Since $0=\widetilde{\rho}(y)=f \cdot \widetilde{\rho}(x)$ we have that $\widetilde{\rho}(x)=q \cdot\left(1+\chi^{2}+\cdots+\chi^{N-2}\right)$ for some $q \in \mathbb{Q}$. Obviously $\mathbf{t}_{(2)}\left(\eta\left(2^{K} \cdot x\right)\right)=0$. If $M>1$, then we have $\mathbf{t}_{(\text {odd })}\left(\eta\left(2^{K} \cdot x\right)\right)=0$ because

$$
\begin{aligned}
\widetilde{\rho}\left(\left(p_{M}^{N}\right)^{!}\left(2^{K} \cdot x\right)\right) & =2^{K} \cdot q \cdot\left(1+\chi^{2}+\cdots+\chi^{N-2}\right)= \\
& =2^{K} \cdot q \cdot 2^{K-1} \cdot\left(1+\chi+\chi^{2}+\cdots+\chi^{M-1}\right)=0
\end{aligned}
$$

and $\left(p_{M}^{N}\right)^{!}\left(2^{K} \cdot x\right)=0$ since $\widetilde{\rho}$ yields an injective homomorphism of $\mathcal{S}^{s}\left(L_{M}^{4 e-1}\left(\alpha_{k}\right)\right)$. Hence $\eta\left(2^{K} \cdot x\right)=0$ and $2^{K} \cdot x \in \widetilde{L}_{4 e}^{s}(G)$ and hence $2^{K} \cdot q \in \mathbb{Z}$.

We want to show $2^{-l} \cdot q \in \mathbb{Z}$. This is obviously true if $q=0$. If $q \neq 0$ then we can write $q=z \cdot 2^{h}$ with $h, z \in \mathbb{Z}$ and $z$ odd. There exist $z^{\prime}, z^{\prime \prime} \in \mathbb{Z}$ such that $\left(z^{\prime} \cdot z-1\right) \cdot 2^{h}=2^{l} \cdot z^{\prime \prime}$. We obtain

$$
\begin{aligned}
2^{h} \cdot\left(1+\chi^{2}+\cdots+\chi^{N-2}\right) & =z^{\prime} \cdot \widetilde{\rho}(x)-z^{\prime \prime} \cdot \widetilde{\rho}(\nu)= \\
& =\widetilde{\rho}\left(z^{\prime} \cdot x-z^{\prime \prime} \cdot \nu\right) \in \operatorname{im}\left(\widetilde{\rho}: \mathcal{S}^{s}\left(L_{N}^{4 e-1}\left(\alpha_{k}\right)\right) \rightarrow \mathbb{Q} R_{\widehat{G}}\right)
\end{aligned}
$$

which implies $h \geq l$ and $2^{-l} \cdot q=z \cdot 2^{h-l} \in \mathbb{Z}$.

Set $n:=2^{-l} \cdot q \in \mathbb{Z}$. Notice $x-n \cdot \nu \in \mathcal{S}^{s}\left(L_{N}^{4 e-1}\left(\alpha_{k}\right)\right)$ tors because $\widetilde{\rho}(x-n \cdot \nu)=$ $\widetilde{\rho}(x)-n \cdot \widetilde{\rho}(\nu)=0$.

We obtain

$$
\begin{aligned}
y & =\Sigma(x)+n_{\mu} \cdot \mu= \\
& =\Sigma(x-n \cdot \nu)+n \cdot \Sigma(\nu)+n_{\mu} \cdot \mu \in \operatorname{im}\left(\left.\Sigma\right|_{\text {tors }}\right)+\langle\Sigma(\nu)\rangle+\mathbb{Z}_{2}(\mu)
\end{aligned}
$$

A comparison of the orders of the groups leads to $|\langle\Sigma(x)\rangle| \geq 2^{\min \{K, 2 e\}}$. Since $\mathcal{S}^{s}\left(L_{N}^{4 e+1}\left(\alpha_{k}\right)\right)_{\text {tors }}$ has no elements of order larger than $2^{\min \{K, 2 e\}}$, we conclude

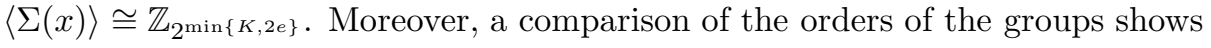

$$
\mathcal{S}^{s}\left(L_{N}^{4 e+1}\left(\alpha_{k}\right)\right)_{\text {tors }}=\operatorname{im}\left(\left.\Sigma\right|_{\text {tors }}\right) \oplus \mathbb{Z}_{2^{\min \{K, 2 e\}}}(\Sigma(\nu)) \oplus \mathbb{Z}_{2}(\mu) .
$$

It remains to show $l=4-\min \{K, 2 e\}$.

On one hand we have $2^{\min \{K, 2 e\}} \cdot \nu \in \operatorname{ker}(\Sigma)$ which implies $2^{\min \{K, 2 e\}} \cdot \nu=m \cdot \omega$ for some $m \in \mathbb{Z}$. Therefore $2^{\min \{K, 2 e\}+l}-m \cdot 2^{4}=0$ and hence $2^{\min \{K, 2 e\}+l-4}=m \in \mathbb{Z}$ and so $l \geq 4-\min \{K, 2 e\}$.

On the other hand let $\Delta:=2^{4-l} \cdot \nu-\omega$. This has $\widetilde{\rho}(\Delta)=0$, so we have $\left.\Delta \in \mathcal{S}^{s}\left(L_{N}^{4 e-1}\left(\alpha_{k}\right)\right)\right|_{\text {tors }}$. Since $\Sigma(\Delta)=2^{4-l} \cdot \Sigma(\nu)$ and $\operatorname{im}\left(\left.\Sigma\right|_{\text {tors }}\right) \cap\langle\Sigma(\nu)\rangle=\{0\}$ we conclude $2^{4-l} \cdot \Sigma(\nu)=0$ and so $4-l \geq \min \{K, 2 e\}$ and $l \leq 4-\min \{K, 2 e\}$.

Remark 7.2. To avoid low-dimensional problems we need to make an ad-hoc definition. Recall that

$$
\mathcal{S}^{s}\left(L_{N}^{5}\left(\alpha_{k}\right)\right)_{\text {tors }} \cong \mathbb{Z}_{2^{\max \{K, 2\}}} \oplus \mathbb{Z}_{2}\left(\mu_{2}\right)
$$


Choose a generator of the first summand and denote it $\mu_{4}$.

Recall from the previous section that we have an isomorphism on the torsion parts $\Sigma: \mathcal{S}^{s}\left(L_{N}^{4 e+1}\left(\alpha_{k}\right)\right)_{\text {tors }} \rightarrow \mathcal{S}^{s}\left(L_{N}^{4 e+3}\left(\alpha_{k}\right)\right)_{\text {tors }}$. Also note that $\nu_{e} \in \mathcal{S}^{s}\left(L^{4 e-1}\left(\alpha_{k}\right)\right)$ in Proposition 7.1 is not unique. Two choices of such $\nu_{e}$ differ by a torsion element. Therefore a direct sum decomposition of $\mathcal{S}^{s}\left(L^{4 e-1}\left(\alpha_{k}\right)\right)$ into the free abelian part and torsion part as in Proposition 4.1 determines $\nu_{e}$ uniquely by demanding that the projection on the torsion part is 0 . So let us suppose that we have such a decomposition for every $e \geq 2$. Then we obtain by induction:

Corollary 7.3. Let $G=\mathbb{Z}_{N}$ with $N=2^{K} \cdot M, M$ odd and $K \geq 1, d \geq 3$. Then we have an isomorphism

$$
\left(\overline{\mathbf{r}}_{4 i}, \overline{\mathbf{r}}_{4 i-2}\right)_{i}: \mathcal{S}^{s}\left(L_{N}^{2 d-1}\left(\alpha_{k}\right)\right)_{\mathrm{tors}} \cong \bigoplus_{i=1}^{c} \mathbb{Z}_{2^{\min \{K, 2 i\}}}\left(\mu_{4 i}\right) \oplus \bigoplus_{i=1}^{c} \mathbb{Z}_{2}\left(\mu_{4 i-2}\right)
$$

where $\mu_{4 i}=\Sigma^{2(c-i)}\left(\nu_{i}\right)$ when $i \geq 2$.

Remark 7.4. The invariants $\overline{\mathbf{r}}_{2 i}$ are defined simply as the factors of the isomorphism which follows by induction from Proposition 7.1. The bar indicates that the invariants $\overline{\mathbf{r}}_{4 i}$ differ from the invariants $\mathbf{r}_{4 i}$ from our main Theorem 1.2, We prefer the new invariants since it follows that they have a geometric interpretation as we describe in more detail in the following remark.

Remark 7.5. The promised interpretation follows directly from Corollary 7.3 In detail, when $i \geq 2$, it follows from the earlier discussion about splitting problems that the invariants

$$
\overline{\mathbf{r}}_{4 i}: \mathcal{S}^{s}\left(L_{N}^{4 e+1}\left(\alpha_{k}\right)\right) \rightarrow \mathbb{Z}_{2^{\min \{K, 2 i\}}}\left(\mu_{4 i}\right)
$$

can be described as follows. Let $h: L(\alpha) \rightarrow L_{N}\left(\alpha_{k}\right)$ be a simple homotopy equivalence which represents $x \in \mathcal{S}^{s}\left(L_{N}^{4 e+1}\left(\alpha_{k}\right)\right)$. Suppose that $\overline{\mathbf{r}}_{2 j}(x)=0$ for $j>i$. Then we know that $h$ is homotopic to a simple homotopy equivalence (still denoted $h$ ) which is split along $L_{N}^{4 i-1}\left(\alpha_{k}\right)$. Let $L_{N}^{4 i-1}(\beta):=h^{-1}\left(L_{N}^{4 i-1}\left(\alpha_{k}\right)\right)$. Then $h \mid: L_{N}^{4 i-1}(\beta) \rightarrow L_{N}^{4 i-1}\left(\alpha_{k}\right)$ represents an element, say $y \in \mathcal{S}^{s}\left(L_{N}^{4 i-1}\left(\alpha_{k}\right)\right)$. Then $\overline{\mathbf{r}}_{4 i}(x)$ is the value of $y$ under the composition

$$
\mathcal{S}^{s}\left(L_{N}^{4 i-1}\left(\alpha_{k}\right)\right) \stackrel{\widetilde{\rho}}{\rightarrow} \mathbb{Q} R_{\widehat{G}} \stackrel{\chi \mapsto-1}{\longrightarrow} \mathbb{Q} \stackrel{: M: 2^{3+\max \{0, K-2 i\}}}{\longrightarrow} \mathbb{Q} .
$$

Notice that this value does not depend on the chosen decomposition of $\mathcal{S}^{s}\left(L^{4 i-1}\left(\alpha_{k}\right)\right)$ into the direct sum of the free abelian part and the torsion part.

Remark 7.6. It follows from the results of the previous section that the invariants $\overline{\mathbf{r}}_{4 i-2}$ also have a geometric description, when $i \geq 2$. Namely, recall that in that case we have $\overline{\mathbf{r}}_{4 i-2}=\mathbf{r}_{4 i-2}=\mathbf{t}_{4 i-2}$ and after transferring via $\left(p_{2}^{N}\right)$ ! (which is an isomorphism on the $\mathbb{Z}_{2}\left(\mu_{4 i-2}\right)$ summands) we have $\mathbf{t}_{4 i-2} \circ \eta=\sigma$, where $\sigma$ is the Browder-Livesay invariant.

Remark 7.7. In the last two sections we have restricted ourselves to the simple structure sets of $L_{N}^{2 d-1}(\alpha)$ where $\alpha=\alpha_{k}$, whereas in the main Theorem 1.2 this restriction is not present. To understand this, recall that we have first obtained the calculation for $L_{N}^{2 d-1}\left(\alpha_{k}\right)$ and then used the fact that for any $\alpha$ there exists $k$ and a homotopy equivalence $h: L_{N}^{2 d-1}(\alpha) \rightarrow L_{N}^{2 d-1}\left(\alpha_{k}\right)$. Such $h$ induces an isomorphism on the simple structure sets. However, this induced isomorphism has no good geometric description. It would certainly be interesting to understand this problem. 


\section{REFERENCES}

[AS68] M. F. Atiyah and I. M. Singer. The index of elliptic operators. III. Ann. of Math. (2), 87:546-604, 1968

[HT00] Ian Hambleton and Laurence R. Taylor. A guide to the calculation of the surgery obstruction groups for finite groups. In Surveys on surgery theory, Vol. 1, volume 145 of Ann. of Math. Stud., pages 225-274. Princeton Univ. Press, Princeton, NJ, 2000.

[LdM71] S. López de Medrano. Involutions on manifolds. Springer-Verlag, New York, 1971. Ergebnisse der Mathematik und ihrer Grenzgebiete, Band 59.

[Mil66] J. Milnor. Whitehead torsion. Bull. Amer. Math. Soc., 72:358-426, 1966.

[MW08] Tibor Macko and Christian Wegner. On fake lens spaces with the fundamental group of order a power of 2. Preprint, arXiv: math.AT., 2008.

[Pet70] Ted Petrie. The Atiyah-Singer invariant, the Wall groups $L_{n}(\pi, 1)$, and the function $\left(t e^{x}+1\right) /\left(t e^{x}-1\right)$. Ann. of Math. (2), 92:174-187, 1970.

[Ran81] Andrew Ranicki. Exact sequences in the algebraic theory of surgery, volume 26 of Mathematical Notes. Princeton University Press, Princeton, N.J., 1981.

[Ran92] A. A. Ranicki. Algebraic L-theory and topological manifolds, volume 102 of Cambridge Tracts in Mathematics. Cambridge University Press, Cambridge, 1992.

[Wal99] C. T. C. Wall. Surgery on compact manifolds, volume 69 of Mathematical Surveys and Monographs. American Mathematical Society, Providence, RI, second edition, 1999. Edited and with a foreword by A. A. Ranicki.

Mathematisches Institut, Universität MÜnster, Einsteinstrasse 62, MÜnster, D-48149, Germany, and Matematický Ústav SAV, Štefánikova 49, Bratislava, SK-81473, Slovakia E-mail address: macko@uni-muenster.de

Mathematisches Institut, Universität Münster, Einsteinstrasse 62, MÜnster, D-48149, GERMANY

E-mail address: c.wegner@uni-muenster.de 